\title{
Deep-Learning-Based Road Crack Detection Frameworks for Dashcam-captured Images under Different Illumination Conditions
}

Da-Ren Chen ( $\sim$ danny@gm.nutc.edu.tw)

National Taichung Institute of Technology: National Taichung University of Science and Technology https://orcid.org/0000-0002-1185-7208

Wei-Min Chiu

National Taichung University of Science and Technology

\section{Research Article}

Keywords: Convolution Neural Network, Gaussian mixture models, Road Crack Detection.

Posted Date: July 13th, 2021

DOI: https://doi.org/10.21203/rs.3.rs-685762/v1

License: (c) (i) This work is licensed under a Creative Commons Attribution 4.0 International License.

Read Full License 


\section{Title Page}

To:

Soft Computing

Editorial Office

Dear Editors,

We submit a new manuscript entitled "Deep-Learning Road Crack Detection for Dashcam-captured Images under Different Illumination Conditions" to Soft Computing.

This work described has not been submitted elsewhere for publication, in whole or in part. To the best of our knowledge, the named authors have no conflict of interest, financial or otherwise. We also have read and have abided by the statement of ethical standards for manuscripts submitted to Soft Computing.

Your sincerely,

Prof. Da-Ren Chen

Department of information management, National Taichung University of Science and Technology,

No.129,Sec.3,Sanmin Rd,North Dist.,Taichung City 404, Taiwan (R.O.C.)

Corresponding author: Da-Ren Chen

Email: danny@ nutc.edu.tw ORCID: 0000-0002-1185-7208

Tel: +886-4-2219-6883, +886-933-205261

Mr. Wei-Min Chiu

Department of information management, National Taichung University of Science and Technology,

No.129,Sec.3,Sanmin Rd,North Dist.,Taichung City 404, Taiwan (R.O.C.) 


\title{
Deep-Learning-Based Road Crack Detection Frameworks for
}

\section{Dashcam-captured Images under Different Illumination}

\section{Conditions}

\begin{abstract}
Machine learning techniques have been used to increase detection accuracy of cracks in road surfaces. Most studies failed to consider variable illumination conditions on the target of interest (ToI), and only focus on detecting the presence or absence of road cracks. This paper proposes a new road crack detection method, IlumiCrack, which integrates Gaussian mixture models (GMM) and object detection CNN models. This work provides the following contributions: 1) For the first time, a large-scale road crack image dataset with a range of illumination conditions (e.g., day and night) is prepared using a dashcam. 2) Based on GMM, experimental evaluations on 2 to 4 levels of brightness are conducted for optimal classification. 3) the IlumiCrack framework is used to integrate state-of-the-art object detecting methods with CNN to classify the road crack images into eight types with high accuracy. Experimental results show that IlumiCrack outperforms the state-of-the-art R-CNN object detection frameworks.
\end{abstract}

Keywords: Convolution Neural Network, Gaussian mixture models, Road Crack Detection.

\section{Declarations}

Funding: This work was supported in part by National Taichung University of science and technology under Grant NTCUST109-13.

Conflicts of interest: I and another author declare that we have no conflict of interest and Competing interests.

\section{Introduction}

Climate factors and heavy-load transportation requirements have left road surfaces in Taiwan vulnerable to damage from natural disasters and daily wear and tear. Road cracks are usually scattered over the road surface, making it difficult to predict potential threats to road safety and vehicles. Traditional road inspection techniques are time and labor-intensive, and many local authorities have begun to outsource this work to the general driving public through telephone or online reporting [9]. Drivers with 
smartphones or dashcams report the damage location and upload images or video of the damage to a central website. Local authority staff then evaluate the images and assign a maintenance priority.

In recent years, dashcams have become popular with drivers, with some models automatically uploading images and video to cloud storage, making them a promising source information for automatic road crack detection.

In the development of traditional image detection techniques for road cracks, researchers empirically tuned a series of filters to differentiate cracks from background noise $[1,2,3,4]$. Notwithstanding significant improvements in image processing-based crack detection methods, such approaches may not work without manually feature adjustment for varying crack patterns. In actual road conditions, these approaches have difficulty adapting to all scenarios due to complex illumination conditions and varying crack shapes or textures [5]. In recent years, researchers have sought to develop solutions by applying machine learning methods.

Deep learning [6,7,8], a branch of machine learning, outperforms traditional image processing and other machine learning techniques [9,10]. First, it simplifies the work flow of machine learning because it learns all features automatically without handcrafted feature selections. Second, the incremental representations of its intermediate levels are learned jointly such that the feedback and parameters for each layer are dedicated to global optimization. Third, it applies multiple nonlinear layers of neural networks to better represent data, leading to better results or models.

In recent years, significant improvements have been made in road crack identification using novel deep learning frameworks such as Region with CNN(R-CNN) [11], You Only Look Once (YOLO) [10], Single Shot multibox Detection (SSD) [12] etc. Zang et al. [11] photographed road cracks using a smart phone, and increased image sample diversity by using random shooting angles. They applied R-CNN to a preprocessed dataset to identify road cracks. Zalama et al. [10] photographed road cracks using a car-based hi-speed camera, and trained the dataset using AdaBoost [14]. Shi et al. [15] identified road cracks using random structured forests in which the dataset are preprocessed jointly with diverse image channels. Maeda et al. [12] collected road cracks through a car-based smart phone and proposed a classification method for the dataset. They applied the object detection framework SSD to train the dataset, obtaining considerable improvements to identification accuracy. Recent studies on road crack detection/identification are compared in Table 1.

Table 1. Recent studies on road crack detection

\begin{tabular}{|c|c|c|}
\hline & Strengths & Weakness \\
\hline $\begin{array}{l}\text { Zhang et al. [11] } \\
(2016)\end{array}$ & $\begin{array}{l}\text { Low cost, smart phone- } \\
\text { based data collection }\end{array}$ & $\begin{array}{l}\text { Considers only crack } \\
\text { patterns } \\
\text { Considers only daylight }\end{array}$ \\
\hline
\end{tabular}




\begin{tabular}{|c|c|c|}
\hline & & conditions \\
\hline $\begin{array}{l}\text { Zalama et } \\
\text { al.[10] (2014) }\end{array}$ & $\begin{array}{l}\text { - Integrates multiple } \\
\text { classifications }\end{array}$ & $\begin{array}{ll} & \text { Considers only crack } \\
\text { patterns } \\
\text { - } \\
\text { High data collection cost } \\
\text { Considers only daylight } \\
\text { conditions }\end{array}$ \\
\hline $\begin{array}{l}\text { Shi et al.[10] } \\
(2016)\end{array}$ & $\begin{array}{l}\text { Uses joint diverse image } \\
\text { channels for } \\
\text { preprocessing } \\
\text { Low cost, smart phone- } \\
\text { based data collection }\end{array}$ & $\begin{array}{l}\text { Considers only crack } \\
\text { patterns } \\
\text { Considers only daylight } \\
\text { conditions }\end{array}$ \\
\hline $\begin{array}{l}\text { Maeda et al.[12] } \\
\text { (2018) }\end{array}$ & $\begin{array}{l}\text { Proposes new } \\
\text { classification for road } \\
\text { cracks } \\
\text { Low cost, smart phone- } \\
\text { bassed data collection }\end{array}$ & $\begin{array}{l}\text { - Considers only daylight } \\
\text { conditions }\end{array}$ \\
\hline
\end{tabular}

As seen in Table 1, most studies do not consider diverse illumination conditions. In addition, with the exception of Maeda et al.[12], the other studies only consider road crack patterns without other road markings such as crosswalks, line dividers etc. In this paper, we first collect additional road datasets during different daylight periods through a low-cost dashcam so as to ensure the dataset better represents a wide variety of authentic road use conditions. Second, we examine the collected datasets to assess the influence of pixel divergence on the state-of-the-art object detection frameworks YOLO and SSD. Third, we study and determine the most appropriate number of categories of pixel brightness to reach the highest detection accuracy. On the basis of GMM, we propose an object detection framework that integrates YOLO, SSD and neural networks to classify dataset images according to brightness features and to identify the location and class of individual road cracks. The performance of the framework is evaluated to determine the optimal number of brightness groups, it also compared to the ordinary YOLO and SSD to understand its outperformance on road crack detection.

The remainder of this paper is organized as follows. Section 2 describes the methodology and state-of-the-art object detection frameworks. Section 3 presents the proposed framework and algorithms. Section 4 describes the experimental setup and results. Finally, Section 5 draws concluding remarks.

\section{Methodology}

\subsection{Gaussian Mixture Model (GMM)}

Stauffer et al [16] applied the Gaussian Mixture Model (GMM) to create a background model that enhances the differentiation between background and foreground in an image. Different from Gaussian distribution, GMM considers the effect of prior probability to derive the probability of its Gaussian distributions and can 
be denoted as

$$
\begin{gathered}
p(x \mid \theta)=\sum_{k=1}^{K} \alpha_{k} N\left(x \mid \mu_{k}, \sum_{k}\right) \\
\sum_{k=1}^{K} \alpha_{k}=1,0 \leq \alpha_{k} \leq 1
\end{gathered}
$$

where $\sum_{k}$ denotes the covariance matrix, and $\mu_{k}$ denotes the average of distribution $\mathrm{k}$ in a GMM model. Notation $\alpha_{k}$ denotes the probability of distribution $\mathrm{k}$ in the model.

In most cases, the parameters are unknown to the GMM model, thus requiring an iterative algorithm to find the best parameter combinations. The expectationmaximization (EM) algorithm [15] (Algorithm 1) finds the maximum likelihood or maximum a posteriori estimates of parameters, depending on unobserved latent variables in the model.

Algorithm $1 \mathrm{GMM}$-EM

Input : dataset $X$

Output : parameters of GMM

Step 1. Initialize $\left\{\left\{\alpha_{k}, \mu_{k}, \sum_{k}\right\} \mid 1 \leq k \leq K\right\}$

Step 2. Derive the likelihood of sample $x_{i}$ in group k using $\gamma(i, k)=\frac{\alpha_{k}^{(t)} N\left(x_{i} \mid \mu_{k}^{(t)}, \Sigma_{k}^{(t)}\right)}{\sum_{j=1}^{K} N\left(x_{i} \mid \mu_{j}^{(t)}, \Sigma_{j}^{(t)}\right)}$

Step 3. Re-estimate $\alpha_{k}^{(t+1)}, \mu_{k}^{(t+1)}, \sum_{k}^{(t+1)}$ using $=\frac{1}{n} \sum_{i} x_{i}$ and $\sum^{2}=\frac{1}{n} \sum_{i}\left(x_{i}-\mu\right)^{2}$

Step 4. Repeat Stage 2 and Stage 3 until the error between iterations $t+1$ and $t$ is less than 0.001

GMM not only provides a smooth overall distribution fit, its components can clearly detail a multimodal density [17]. Gao et al. [16] have concluded that GMM outperforms single modal Gaussian distribution in background and pixel classification. Zeng et al. [18] proposed a GMM-based color reduction algorithm that classifies images with a pixel color quantization representation. In this paper, the motivation to apply GMM to the pixel identification problem is that the GMM has been shown to be a powerful approach to text-independent speech verification and identification which bears a strongly similarity to the task of illumination and texture classification for images.

\subsection{Convolution Neural Network (CNN)}

A Convolutional Neural Network $(\mathrm{CNN})$ is a deep learning algorithm first proposed by LeCun et al [7]. CNN is widely used in image processing, where the regional features 
are convolved with the kernel to generate new outputs. Unlike traditional image processing methods where the kernel parameters are predefined, the parameters in convolutional layers are determined during the training process. CNN identifies the obvious features in the images using a convolution layer, reduces the image size in a pooling layer, and resolves linear classification through a fully-connected layer with activation functions.
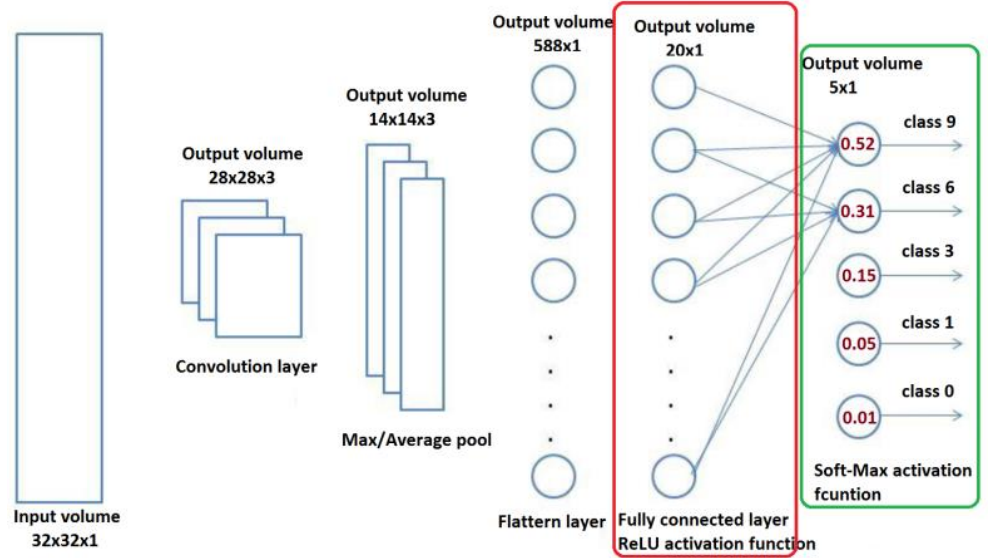

Fig. 1. Convolution Neural Network (CNN) structure

Using sliding window and convolution operations, the convolution layer shown in Fig.1 assigns levels of importance through a predefined filter to various aspects or objects in the image, and differentiates them from each other. These operations extract the high-level features, such as edges. from the input image, analogous to the connectivity pattern of neurons in the human brain. Conventionally, the first layer captures the low-level features such as edges, color, gradient orientation, etc. With additional layers, CNN adapts to the high-level features as well, giving us a network which has a holistic understanding of the dataset images, similar to how the human brain processes images.

The pooling layer is used to decrease computational time required to process the data through dimension reduction. It also extracts dominant features which are positional and rotational invariant, thus ensuring effective model training. Max pooling returns the maximum value from a part of the image covered by the kernel. It mostly removes the noisy activations and also performs de-noising along with dimensionality reduction. Average pooling returns the average of all the values from a part of the image covered by the kernel. It performs dimensionality reduction merely as a noise suppressing mechanism.

After converting the input image into a fitting form for the multi-level perceptron, the image will be flattened into a column vector through the flatten layer. The flattened output is then fed to a feed-forward neural network with backpropagation applied to each training iteration. The fully connected layer shown in the right-hand-side of Fig. 1 , is used to learn non-linear combinations of the high-level features as represented by 
the output of the convolutional layer. Over a series of epochs, the model can distinguish between dominating and certain low-level features in images and classify them using a Soft-max activation function. Soft-max, known as the normalized exponential function, is a non-linear function which accelerates the training process and features complex models required in real-life [21]. In particular, it normalizes the multiple outputs of neurons into an interval between 0 and 1 , which can be regarded as probability values over multiple classes. For example, output vector $[9,6,3,1,0]$ from neurons correspond to the values $[0.5294,0.3157,0.1578,0.0526 .0 .01342]$ of the soft-max function.

\subsection{CNN-based object detection frameworks}

Girshich et al. [19] proposed an R-CNN framework with processing stages. In the first stage, selective search [20] is applied to identify and crop 2000 regions of equal size for each of the input images. The second stage performs CNN on those regions and outputs their features in vector form. Finally, a linear SVM is applied to classify those features. However, R-CNN could be time consuming because it requires more crops, leading to considerable redundant computation and memory space consumption from overlapping crops. Fast R-CNN resolves this time consuming and space redundancy problem by using a feature extractor for the entire image so that crops share the computation load of feature extraction. Compared with R-CNN, the framework of Fast R-CNN applies an ROI pooling layer analogous to Max pooling that compresses feature crops into $7 \times 7$ images which accelerates the processing of the full-connected layer. In addition, Fast R-CNN uses two different fully-connected layers. The first is a soft-max regression that predicts the object classification, and the second layer uses regression to predict the location of each object. The selective search is independent of Fast R$\mathrm{CNN}$ and is also time consuming. Faster R-CNN proposed, by Ren et al. [23], remedies this performance issue by replacing selective search with a feature extractor called the Region Proposal Network (RPN) technique. However, Faster R-CNN cannot crop proposals directly from the image and re-runs crops through the feature extractor, leading to redundant computations. 


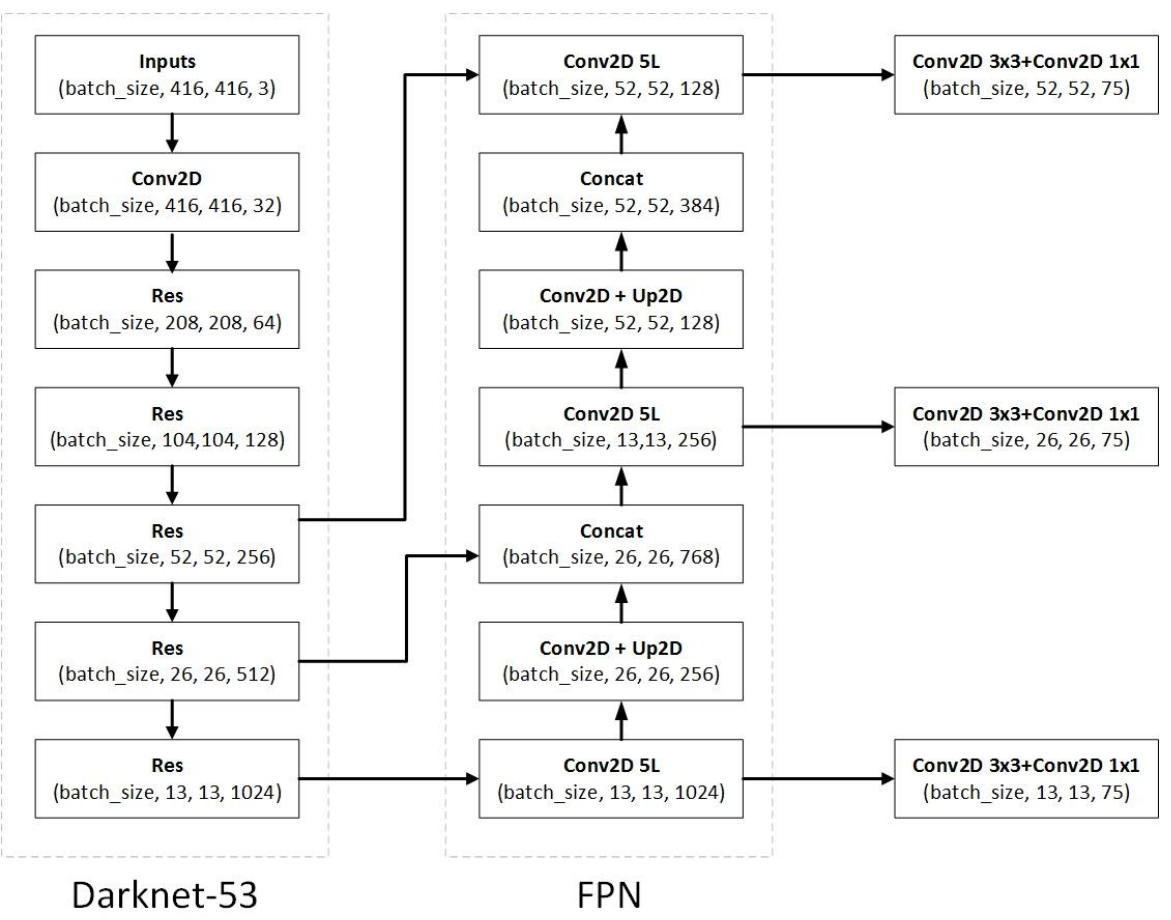

Fig. 2. Framework of YOLO v3 network

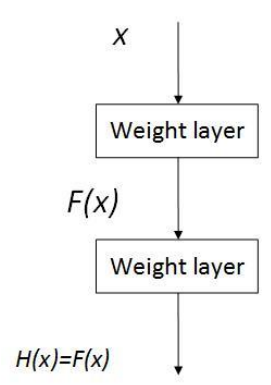

(a)

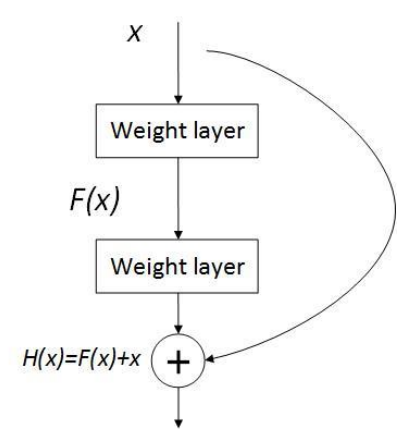

(b)

Fig. 3. A comparison of residual block with shortcut

YOLO is an object detection framework that can predict the region and class of objects with a single CNN. YOLO achieves considerable mean average precision as well as processing speed because background information and regression are involved. The YOLO algorithm produces the locations of the bounding box of the object candidate and the confidence of the inference. To date, YOLO has published three major versions. YOLO v1, announced in 2016 [24], reduces the input images to $448 \times 448$ pixels inputs to the neural network, producing the probabilities and locations of object bounding boxes, filtering the duplicated bounding boxes using Non-Maximum Suppression (NMS), and identifying their final locations. YOLO achieves object detection speeds of up to 45fps on Titan X GPUs. In 2017, YOLO v2 [25] improved on the first version by replacing its fully-connected layers with convolution layers and introduced the notion of an anchor box, inspired by Faster R-CNN. YOLO v3, announced in 2018 [26], is composed of Darknet-53 [24] and Feature Pyramid Network 
(FPN) [27] (Fig. 2), and detects the shrunken input images with scales of 1/32, 1/16 and 1/8. Darknet-53 is applied for feature extraction and improves gradient descent using ResNet [28], proposed by He et al., because a deeper neural network has been shown to not necessarily provide improved precision. Instead, a deeper neural network may incur a vanishing gradient or gradient exploding problem. Therefore, YOLO v3 modifies the VGG19 network with the notion of a "shortcut" shown in Fig. 3. Fig. 3(a) describes an ordinary neural network while Fig. 3(b) shows a neural network with an output residual block denoted as $\mathrm{H}(\mathrm{x})=\mathrm{F}(\mathrm{x})+\mathrm{x}$. When a residual $\mathrm{F}(\mathrm{x})$ approximates 0 during a reverse-propagation stage, YOLO v3 skips this layer using the shortcut. FPN has been applied to road crack detection [27]. Figure 4(a) shows how an image is detected using single scale applied to Fast R-CNN and Faster R-CNN. Prediction rates fall when input image sizes are non-uniform. In Fig. 4(b), the SSD object detection framework detects images with different scales. Figure 4(c) presents the principle of FPN which extracts the features from different image scales in a bottom-up fashion, while enhancing these image scale features in a top-down fashion.

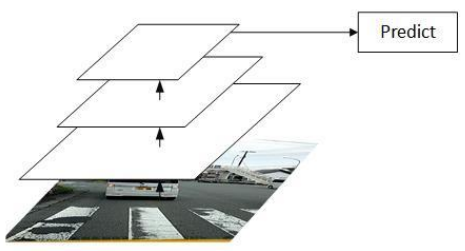

(a)Single feature map

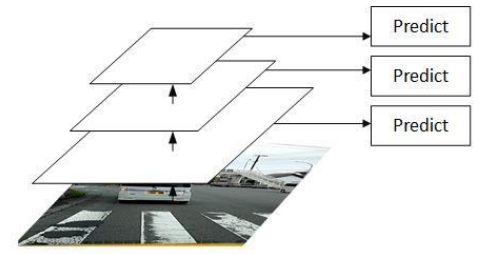

(b)Pyramidal feature hierarchy

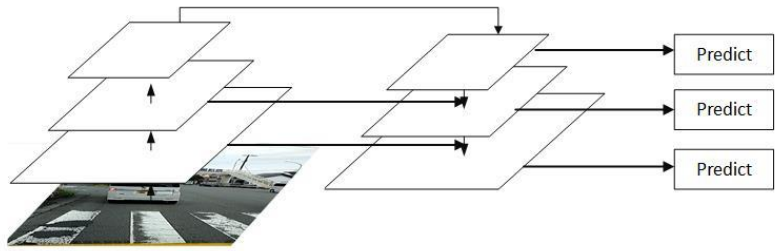

(c)Feature Pyramidal Network

Fig. 4. Comparison of object detections

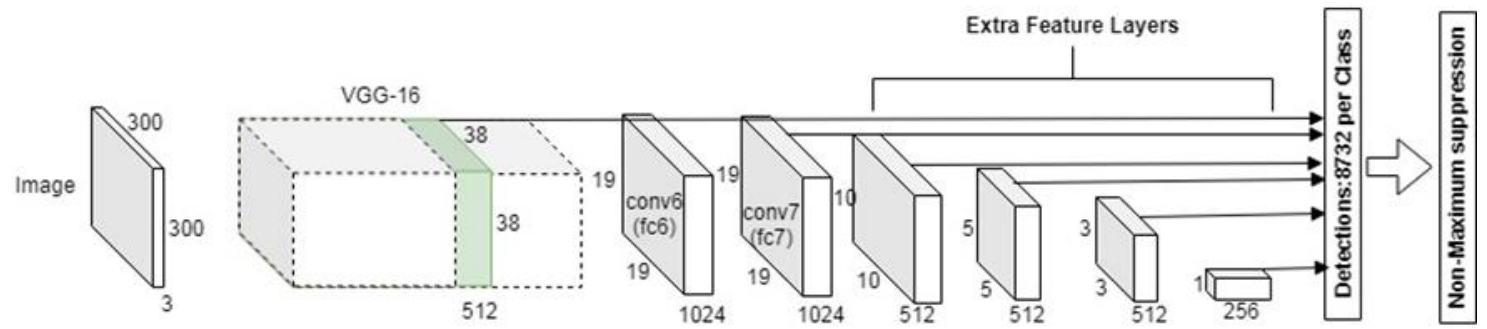

Fig. 5. Overview of SSD framework

Proposed by Liu et al. in 2016 [29], SSD adopts the feature extractor VGG-16 [30]. Similar to YOLO, SSD runs a CNN on the input image only once to learn the representations. A series of $3 \times 3$ convolution filters are performed on these representations to predict the bounding boxes and class probability. The key feature of 
SSD is its use of pyramid feature hierarchy to predict objects with different scales after using multiple convolutional layers. Figure 5 presents an overview of the SSD framework, where fully-connected layers fc6 and fc7 are replaced with convolution layers conv6 and conv7 such that the SSD expands the coverage of convolution detection without additional parameters or complex models [31]. Due to the multi-scale property, SSD produces 8732 default boxes for each class according to the convolution layers with different aspect ratios. Using the non-maximum suppression (NMS) technique [32], SSD filter out default boxes with low probabilities. The example shown in Fig. 6 illustrates two objects with four default boxes on the top-left corner. Each default box is scored and sorted in descending order. The default box with the highest score is computed with other default boxes using the Intersection Over Union (IoU) operation. When the IoU of those boxes exceeds a certain threshold, the boxes with lower confidence scores are set to 0 , otherwise the box is not relevant to the highest score box. The default box with the highest score is labeled with a select object. The NMS algorithm is performed until all default boxes score less than 0 .

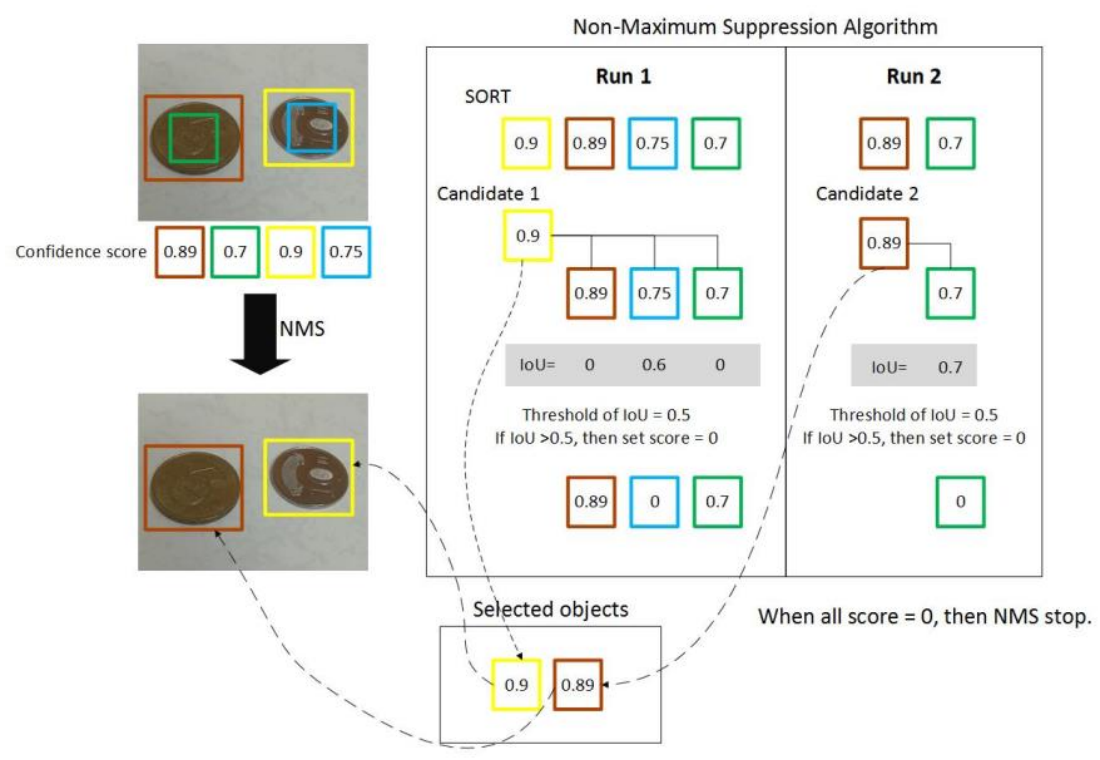

Fig. 6. Example of non-maximum suppression algorithm

\section{Proposed Method}

The proposed framework shown in Fig. 7 is composed of classification and training phases. In the classification phase, a large number of road crack images are gathered over a single day. Those images are formulated as vectors according to HSV (hue, saturation, value) representations and processed with dimensionality reduction. Before the training phase, the dataset is classified in terms of pixel HSV and image features. Sections 3.2 and 3.3 respectively discuss the effectiveness of pixel and image classification, along with how many groups of pixels/images would 
contribute to the best precision rate.

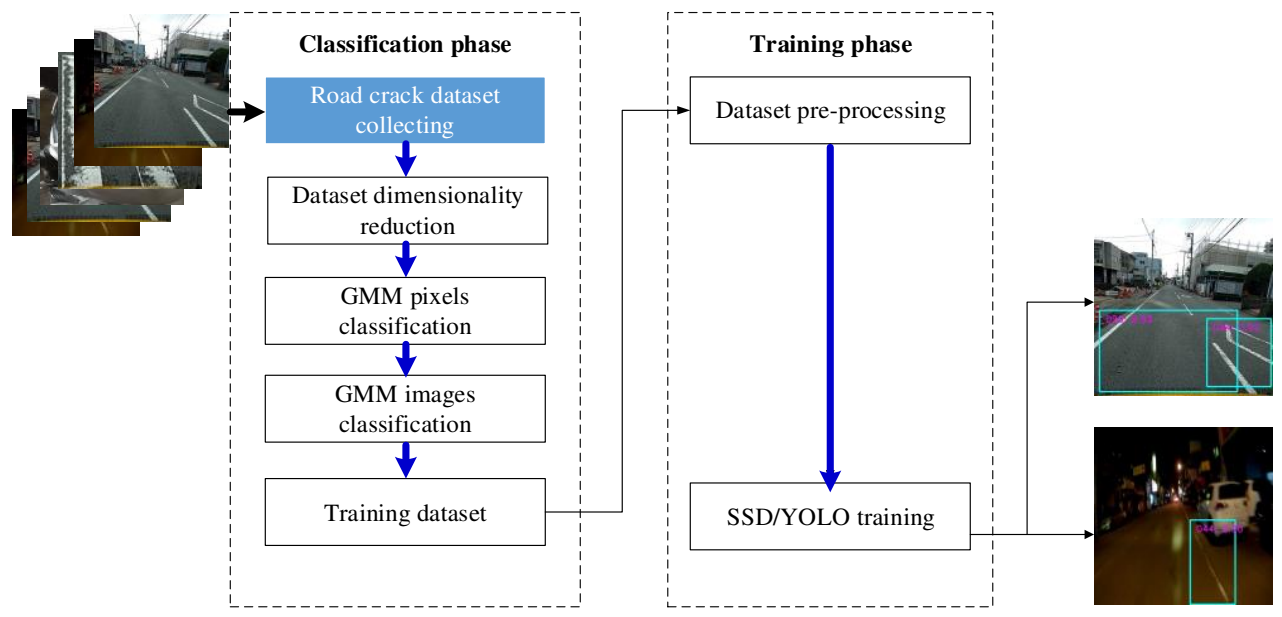

Fig. 7. Road crack detection framework

\subsection{Dataset dimensionality reduction}

HSV has been applied to classify an image as daytime or nighttime images [33]. Hue and value thresholds are set to group images into daytime and nighttime images. Taha et al. [34] applied this technique to characterize cracks as being portrayed in daytime or nighttime images, and to isolate them from noise. Assume $X$ denotes the dataset of road images and can be defined in Eq. (1)

$$
X=\left[\begin{array}{cccc}
x_{11} & x_{12} & \cdots & x_{1 m} \\
x_{21} & x_{22} & \cdots & x_{2 m} \\
\vdots & \vdots & \ddots & \vdots \\
x_{d 1} & x_{d 2} & \cdots & x_{d m}
\end{array}\right] \in \mathbb{R}^{d \times m}, x_{i j}=\left(H_{i j}, S_{i j}, V_{i j}\right)^{T},
$$

where $d$ denotes the number of images and $m$ denotes the number of pixels. Variable $x_{i j}$ presents the HSV value of the $j$-th pixel in $i$-th image where $\mathrm{H}, \mathrm{S}$ and $\mathrm{V}$ respectively denote the pixel's hue, saturation and value. To consolidate the image and pixel dimensions while preserving their HSV [31], Eq. (1) can be rewritten as

$$
X=\left[\begin{array}{llll}
x_{1} & x_{2} & \cdots & x_{(d * m)}
\end{array}\right] \in \mathbb{R}^{(d \times m)}, x_{i}=\left(H_{i j}, S_{i j}, V_{i j}\right)^{T} .
$$

\subsection{Pixels classification}

To understand how many groups of pixels substantially improves object detection, the number of groups of pixel HSV are given as $P=\{2,3,4\}$. Based on HSV values, the image pixels can be denoted as a Gaussian distribution $N\left(x \mid \mu_{p}, \sum_{p}\right)$ as shown in Eq. (3),

$$
p(x \mid \theta)=\sum_{p=1}^{P} \alpha_{p} N\left(x \mid \mu_{p}, \sum_{p}\right),
$$

where $\theta$ denotes the parameter. When the dataset is divided into $p$ groups, $\alpha_{p}$ denotes the probability of the individual pixel group, $\mu_{p}$ is the reference HSV, and $\sum_{p}$ is the pixel distribution. The sum of probability of the pixel groups is 1 .

$$
\sum_{p=1}^{P} \alpha_{p}=1,0 \leq \alpha_{p} \leq 1,
$$

To find satisfactory parameters in GMM, the EM algorithm is applied with initialized 
parameters shown in Algorithm Pixel Classification. In Step 2, the pixel values $x_{p}$ are substituted into Eq. (5), deriving the probability of pixel $x_{j}$ in each group. In Step 3, set $x_{i}=\gamma(i, k)$, we derive new values of $\alpha_{p}, \mu_{p}$, and $\sum_{p}$ by substituting $\mu$ and $\sigma^{2}$ derived from Eqs. (6) and (7) for Eq. (5). The process will repeat until error improvement falls below a predefined threshold.

$$
\begin{gathered}
\gamma(j, p)=\frac{\alpha_{p}^{(t)} N\left(x_{j} \mid \mu_{p}^{(t)}, \Sigma_{p}^{(t)}\right)}{\sum_{b=1}^{P} N\left(x_{j} \mid \mu_{b}^{(t)}, \Sigma_{b}^{(t)}\right)} \\
\mu=\frac{1}{n} \sum_{j} x_{j} \\
\sigma^{2}=\frac{1}{n} \sum_{j}\left(x_{j}-\mu\right)^{2}
\end{gathered}
$$

Algorithm Pixel Classification

Input: $X=\left[\begin{array}{llll}x_{1} & x_{2} & \cdots & x_{(d * m)}\end{array}\right] \in \mathbb{R}^{(d \times m)}, x_{i}=\left(H_{i j}, S_{i j}, V_{i j}\right)^{T}$

Output : GMM classification model

Step 1. Initialized parameters $\left\{\left\{\alpha_{p}, \mu_{p}, \sum_{p}\right\} \mid 1 \leq p \leq P\right\}, P=\{2,3,4\}$

Step 2. Compute probability $\gamma(i, k)$ of $x_{i}$ being classified to group $\mathrm{k}$

Step 3. Let $x_{i}=\gamma(i, k)$, re-estimate the values of $\alpha_{p}, \mu_{p}$ and $\sum_{p}$

Step 4. Repeat Step 2 and Step 3, until the difference between the errors of iteration $\mathrm{t}+1$ and $\mathrm{t}$ is less than 0.001

\subsection{Image classification}

After classifying the pixels in accordance with HVS values into 2, 3, and 4 categories, images can be classified in terms of different proportions of pixel categories. To perform image classification, an image's pixel proportions should be referred to by pixel properties. By using one-hot-encoding, the pixel proportions of an image can be formulated by

$$
\begin{gathered}
\text { Image }_{\text {image_ratio }}=\sum_{\text {pixel }}^{\text {image }} \text { oneHotEncoding(GMM_Pixel(pixel)) } \\
p(x \mid \theta)=\sum_{i=1}^{I} \alpha_{i} N\left(x \mid \mu_{i}, \Sigma_{i}\right) \\
\sum_{i=1}^{I} \alpha_{i}=1,0 \leq \alpha_{i} \leq 1 .
\end{gathered}
$$

In addition to discussing the effectiveness of the number of clusters on the $\mathrm{CNN}$ object detection framework, the number of groups in pixel HSV are predetermined in $I=\{2,3$, 4 \}. As shown in Eq. (9), an image is composed of the pixels of $(2,3$, or 4) groups, where $\theta$ denotes the parameters of GMM, $\alpha_{i}$ defined in Eq. (10) is the probability of individual distribution, $\mu_{i}$ is the average of group $i$, and $\sum_{i}$ is the variance of group $i$. To find the $\theta$ for image classification, the EM algorithm is applied to Algorithm Image 
Classification with input images and a predetermined number of categories in $K$. Step 2 derives the value of $\gamma(i, k)$ denoting the probabilities of image $x_{i}$ belonging to pixel proportion $k(k<K)$ by substituting parameters $\alpha_{k}, \mu_{k}$ and $\sum_{k}$ in Eq. (10). In the Step 3, set $\gamma(i, k)=x_{i}$, the new values of $\alpha_{k}, \mu_{k}$ and $\sum_{k}$ are derived from Eq. (5) by substituting $\mu$ and $\sigma^{2}$ from Eqs. (6) and (7). Repeat Steps 2 and 3 until the difference between $\mathrm{t}$ and $\mathrm{t}+1$ error converges within 0.001 .

Algorithm Image Classification

Input: $X=\left[\begin{array}{llll}x_{1} & x_{2} & \cdots & x_{d}\end{array}\right] \in \mathbb{R}^{d}, x_{i}=$ Image $_{\text {bright_ratio }}$

Output: GMM classification model

Step 1. Initialize parameters $\left\{\left\{\alpha_{k}, \mu_{k}, \sum_{k}\right\} \mid 1 \leq k \leq K\right\}, K=\{2,3,4\}$

Step 2. Compute probability $\gamma(i, k)$ of image $x_{i}$ belonging to group $\mathrm{k}$ of image proportion Step 3. Set $\gamma(i, k)=x_{i}$, re-estimate the values of $\alpha_{p}, \mu_{p}$ and $\sum_{p}$

Step 4. Repeat Step 2 and Step 3, until the difference between the errors of iteration $t+1$ and $t$ is less than 0.001

\subsection{Framework setting}

YOLO applies an input size of $416 \times 416 \times 3$ with momentum of 0.9 to reduce the possibility of local optimization. The learning rate is initialized to 0.001 and is decayed $1 / 10$ when it performs over 400,000 steps, and decayed 1/10 again when it reaches 450,000 steps. Higher learning rates benefits training speed, and decreasing learning rates over time increases detection precision. Due to the limited GPU memory size, the batch size is set to 8 , i.e. obtaining eight random samples once from the training set.

\begin{tabular}{|c|c|c|c|c|}
\hline & Type & Filters & Size & Output \\
\hline \multirow{5}{*}{$1 \mathrm{x}$} & Convolutional & 32 & $3 \times 3$ & $416 \times 416$ \\
\hline & Convolutional & 64 & $3 \times 3 / 2$ & $208 \times 208$ \\
\hline & Convolutional & 32 & $1 \times 1$ & \\
\hline & Convolutional & 64 & $3 \times 3$ & \\
\hline & Residual & & & $208 \times 208$ \\
\hline \multirow{4}{*}{$2 \times$} & Convolutional & 128 & $3 \times 3 / 2$ & $104 \times 104$ \\
\hline & Convolutional & 64 & $1 \times 1$ & \\
\hline & Convolutional & 128 & $3 \times 3$ & \\
\hline & Residual & & & $104 \times 104$ \\
\hline \multirow{4}{*}{$8 x$} & Convolutional & 256 & $3 \times 3 / 2$ & $52 \times 52$ \\
\hline & Convolutional & 128 & $1 \times 1$ & \\
\hline & Convolutional & 256 & $3 \times 3$ & \\
\hline & Residual & & & $52 \times 52$ \\
\hline \multirow{4}{*}{$8 x$} & Convolutional & 512 & $3 \times 3 / 2$ & $26 \times 26$ \\
\hline & Convolutional & 128 & $1 \times 1$ & \\
\hline & Convolutional & 256 & $3 \times 3$ & \\
\hline & Residual & & & $26 \times 26$ \\
\hline \multirow{4}{*}{$4 x$} & Convolutional & 1024 & $3 \times 3 / 2$ & $13 \times 13$ \\
\hline & Convolutional & 512 & $1 \times 1$ & \\
\hline & Convolutional & 1024 & $3 \times 3$ & \\
\hline & Residual & & & $13 \times 13$ \\
\hline
\end{tabular}

Fig. 8. The architecture of darknet-53 in YOLO v3 
Figure 8 illustrates the architecture darknet-53 used by YOLO v3, where Convolutional and Residual respectively denote the convolutional and residual layers. A $3 \times 3$ convolutional filter benefits network depth and decreases the number of parameters while increasing valuable features [30]. Instead of a pooling layer, YOLO v3 uses a $3 \times 3$ convolution layer with a stride of 2 to improve feature loss. A $1 \times 1$ convolutional layer aims to synthesize features instead of convolutional computation. The activation function $f(x)=\max (a x, x)$ behind a convolution layer is Leaky ReLU so that the weighs can be still updated when $x<0$ during the reverse propagation phase.

\begin{tabular}{|c|c|c|c|}
\hline Type & Filters & Size & Output \\
\hline Convolutional & 64 & $3 \times 3$ & $300 \times 300$ \\
\hline Convolutional & 64 & $3 \times 3$ & $300 \times 300$ \\
\hline Maxpool & 64 & $2 \times 2 / 2$ & $150 \times 150$ \\
\hline Convolutional & 128 & $3 \times 3$ & $150 \times 150$ \\
\hline Convolutional & 128 & $3 \times 3$ & $150 \times 150$ \\
\hline Maxpool & 128 & $2 \times 2 / 2$ & $75 \times 75$ \\
\hline Convolutional & 256 & $3 \times 3$ & $75 \times 75$ \\
\hline Convolutional & 256 & $3 \times 3$ & $75 \times 75$ \\
\hline Convolutional & 256 & $3 \times 3$ & $75 \times 75$ \\
\hline Maxpool & 256 & $2 \times 2 / 2$ & $38 \times 38$ \\
\hline Convolutional & 512 & $3 \times 3$ & $38 \times 38$ \\
\hline Convolutional & 512 & $3 \times 3$ & $38 \times 38$ \\
\hline Convolutional & 512 & $3 \times 3$ & $38 \times 38$ \\
\hline Maxpool & 512 & $2 \times 2 / 2$ & $19 \times 19$ \\
\hline Convolutional & 512 & $3 \times 3$ & $19 \times 19$ \\
\hline Convolutional & 512 & $3 \times 3$ & $19 \times 19$ \\
\hline Convolutional & 512 & $3 \times 3$ & $19 \times 19$ \\
\hline Maxpool & 512 & $3 \times 3$ & $19 \times 19$ \\
\hline Convolutional & 1024 & $3 \times 3 / 2$ & $19 \times 19$ \\
\hline Convolutional & 1024 & $1 \times 1$ & $19 \times 19$ \\
\hline Convolutional & 256 & $1 \times 1$ & $19 \times 19$ \\
\hline Convolutional & 512 & $3 \times 3$ & $10 \times 10$ \\
\hline Convolutional & 128 & $1 \times 1$ & $10 \times 10$ \\
\hline Convolutional & 256 & $3 \times 3$ & $5 \times 5$ \\
\hline Convolutional & 128 & $1 \times 1$ & $5 \times 5$ \\
\hline Convolutional & 256 & $3 \times 3$ & $3 \times 3$ \\
\hline Convolutional & 128 & $1 \times 1$ & $3 \times 3$ \\
\hline Convolutional & 256 & $3 \times 3$ & $1 \times 1$ \\
\hline
\end{tabular}

Fig. 9. The architecture of VGG in SSD

The input to the SSD network has a size of $300 \times 300 \times 3$ with momentum of 0.9 , learning rate of 0.001 and batch size of 8 at random. Figure 9 shows the architecture VGG consists of a majority of $3 \times 3$ fully connected layers. Unlike YOLO v3, we scales SSD pictures down by applying maxpool with a filter size of 2 and a stride of 2 (Liu et al., 2016). In addition to the ordinary SSD shown in Fig. 5, we modified the SSD to include convolution layers after the last maxpool layer. Without changing the output size, the filter and padding size of those convolution layers are respectively set to 3 and 1 to further reduce information redundancy. The convolutional layer next to the last maxpool layer has a padding of 6 to produce various image scales for the subsequent convolutional layers. 


\section{Experiments}

\subsection{Data Preparation}

This section describes the acquisition of our dataset, including how it was organized, produced and tagged, along with a description of its contents.

In most previous studies on road crack detection, images are usually simplified or emphasized the objects of interest from the local-trimmed pictures. Models trained using these images are difficult to implement in real-life applications due to the difficulty of producing them. Authentic road images taken from a vehicle-based camera (e.g., dashcams) can, be used to train models for use in practical situations. The widespread use of dashcams or smartphones in cars allows any driver to easily upload captured images to models running in cloud computing environments, or even running natively on stand-alone mobile devices. Similar to the categories in proposed in [12], the damage types described in Table 1 are divided into eight categories which are separated into cracks and other defects. The cracks are divided into linear cracks and alligator cracks. The defects include not only pot holes and rutting, but also blurred road markings.

Table 2. Classes and sample images from the dataset [12]

Class ID Class name
Longitudinal crack
D00 Sample image
D01
Longitudinal construction joint
D10
Lateral crack



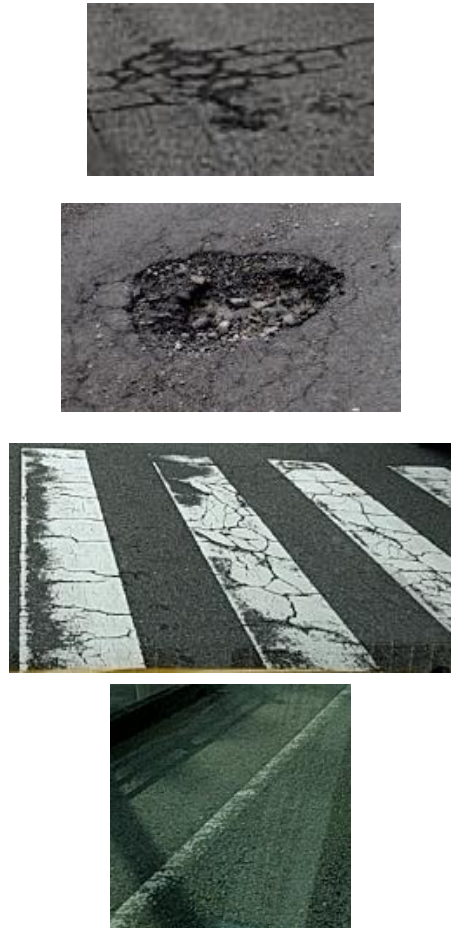

Table 2. Dataset sources

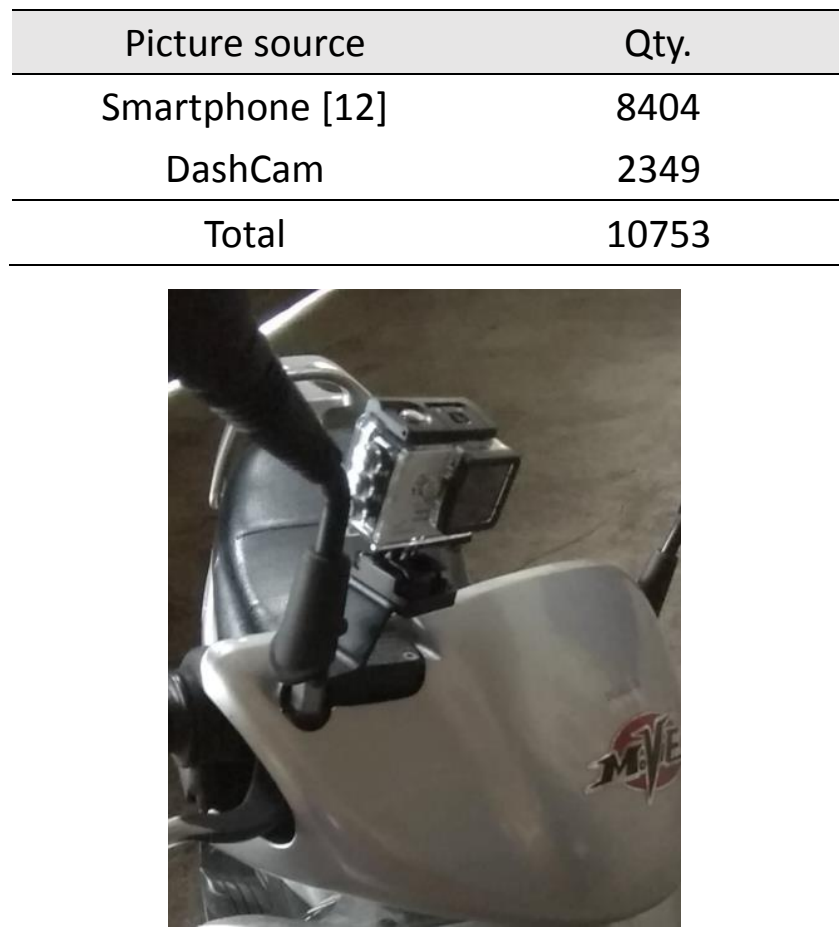

Fig. 10. Dashcam installation setup 


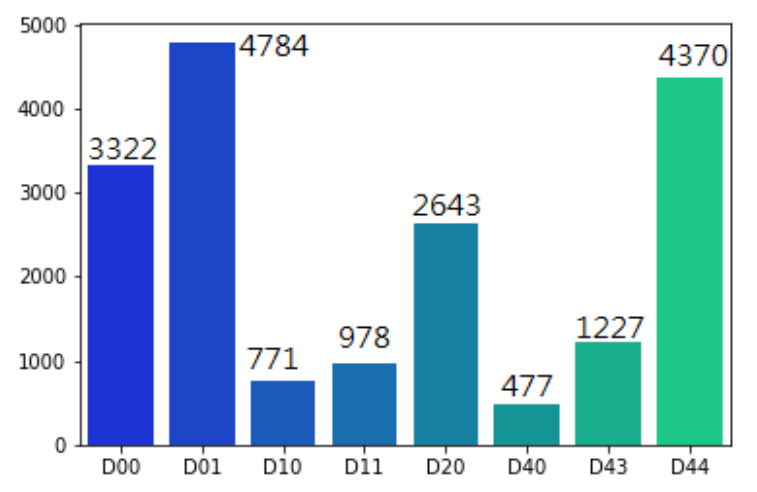

Fig. 11. Tagged image proportions

Images used in this paper were obtained from two sources. First, we reviewed 163,664 smartphone-captured road images from [12], of which 8404 contain cracks. The 8404 images were tagged with the class labels defined in Table 1. Second, because the images obtained from [12] are all taken under daytime lighting conditions, we installed a dashcam (SJCAM SJ5000) on the dashboard of a motorcycle (see Fig. 10), which recorded video as the motorcycle was driven around city streets. Still images measuring 600 x 600 pixels were later extracted from the video files. The sources of tagged picture listed in Table 2 are mixed as a dataset. Images derived from [12] were tagged manually and the proportion of each category is shown in Fig. 11.

During image preprocessing, pictures were transformed to floating point. To enhance model capabilities, the values were duplicated and modified by methods including distortion, expanding, trimming and rotation. Eventually, those pictures were resized to $300 \times 300$. For this purpose, an application that can process road images was developed and is publicly available on our website. All experiments were performed using an Intel Core i5-7400 3.00 GHz CPU with 16GB DDR4 RAM and NVidia GeForce GTX 1080 GPU. The proposed framework was constructed using Python 3.6.8 with a Pytorch neuro network. In our experiment, the dataset was randomly divided in a ratio of $7: 3$, i.e., $70 \%$ of the dataset is training data while $30 \%$ of the dataset is test data.

\subsection{Pixel difference with HSV value}

Table 3. List of clusters

\begin{tabular}{cc}
\hline Qty. of clusters(K) & Cluster ID list \\
\hline 2 & $P D 2-1$, PD2-2 \\
\hline 3 & $P D 3-1$, PD3-2, PD3-3 \\
\hline 4 & $P D 4-1, P D 4-2$, PD4-3 \\
\hline
\end{tabular}

As mentioned Section 3.2, one of the most important goals of this paper is to discuss 
the effectiveness of illumination on a picture in terms of a pixel's HSV value. We also wonder how many categories of HSV are required for optimal results. Following Algorithm Image Classification, the number of categories $k \in \mathrm{K}=\{2,3,4\}$ are investigated. Notation PD $k-n$ denotes the Pixel Difference in k classes where $n$ denotes the $n$-th class shown in Table 3. The clustering results are shown in Fig. 12.

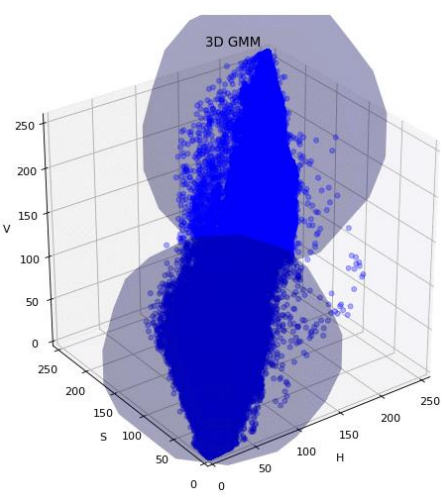

(a)

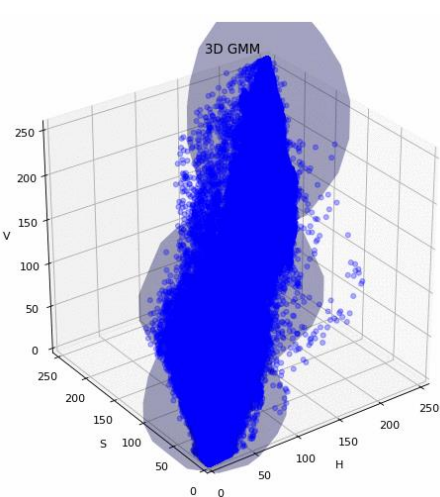

(b)

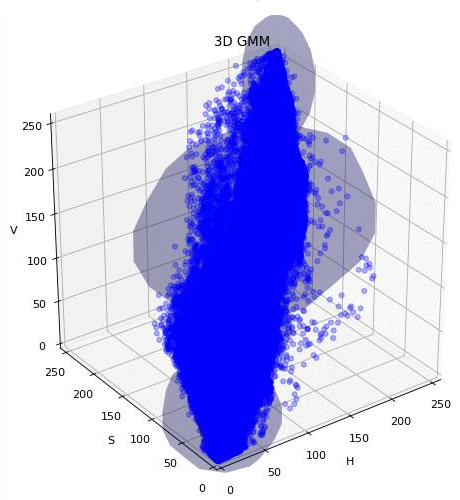

(c)

Fig. 12. Example clustering based on (a) two (b) three and (c) four categories

Figures 12(a), (b) and (c) respectively show examples of clustering with the pixel difference dividing the dataset into two, three and four categories. The circled regions in each figure represent a GMM model and each point denotes a pixel with an X-Y-Z coordinate corresponding to respective values of Hue, Saturation and Value. Abundant cluster point overlap (e.g., the overlap between the two lower clusters in Fig. 12(b)) may interfere with image brightness assessment. When a cluster blurred, it becomes an insignificant factor for object detection.

\subsection{Performance evaluations}

Average precision (AP), a popular metric for measuring the accuracy of object detectors, was first introduced by Everingham et al. [35] in the 2010 PASCAL Visual Object Classes (VOC) competition. AP computes the average precision value for recall value over 0 to 1 on the basis of Eqs. (11) and (12). Precision measures prediction accuracy, i.e., the percentage of correct predictions, and the Recall rate finds the percentage of possible positive cases in our top $\mathrm{N}$ predictions. This section compares the performance of the SSD improved in [12] and YOLO v3 to the proposed framework mentioned in Section 3. Experimental results shown in Table 5 show that, when $\mathrm{K}=2$, the proposed method outperforms SSD except for the images in category D11 because D11 analogues to D10 and increases difficulty using manual data annotation. In addition, the proposed method with $\mathrm{K}=2$ also outperforms SSD and YOLO with $\mathrm{K}>2$. That is, two categories of HVS provide the best precision rate.

$$
A P=\frac{1}{11} \sum_{r \in\{0,0.1, \ldots, 1\}} p_{\text {interp }}(r)
$$




$$
p_{\text {interp }}(r)=\max _{\tilde{r}: \tilde{r} \geq r} p(\tilde{r})
$$

Table 5. Mean AP (mAP) of the target methods

\begin{tabular}{|c|c|c|c|c|c|c|c|c|}
\hline Algorithms & D00 & D01 & D10 & D11 & D20 & D40 & D43 & D44 \\
\hline SSD & 0.46 & 0.61 & 0.10 & 0.11 & 0.67 & 0.18 & 0.83 & 0.71 \\
\hline \multicolumn{9}{|l|}{$K=2$} \\
\hline IlumiCrack(PD2-1)+SSD & 0.67 & 0.79 & 0.19 & 0.09 & 0.83 & 0.39 & 0.85 & 0.82 \\
\hline IlumiCrack(PD2-2)+SSD & 0.63 & 0.60 & 0.56 & 0.29 & 0.83 & 0.53 & 0.90 & 0.86 \\
\hline IlumiCrack(PD2-1)+YOLO & 0.41 & 0.68 & 0.25 & 0.16 & 0.61 & 0.06 & 0.80 & 0.72 \\
\hline IlumiCrack(PD2-2)+YOLO & 0.52 & 0.64 & 0.29 & 0.56 & 0.52 & 0.47 & 0.73 & 0.69 \\
\hline \multicolumn{9}{|l|}{$K=3$} \\
\hline IlumiCrack(PD3-1)+SSD & 0.05 & 0.48 & 0.11 & 0.003 & 0.49 & 0.0 & 0.55 & 0.25 \\
\hline IlumiCrack(PD3-2)+SSD & 0.40 & 0.62 & 0.21 & 0.19 & 0.63 & 0.32 & 0.77 & 0.75 \\
\hline IlumiCrack(PD3-3)+SSD & 0.47 & 0.66 & 0.06 & 0.004 & 0.66 & 0.11 & 0.81 & 0.66 \\
\hline IlumiCrack(PD3-1)+YOLO & 0.24 & 0.65 & 0.28 & 0.22 & 0.43 & 0.0 & 0.60 & 0.30 \\
\hline IlumiCrack(PD3-2)+YOLO & 0.39 & 0.63 & 0.19 & 0.30 & 0.51 & 0.29 & 0.78 & 0.70 \\
\hline IlumiCrack(PD3-3)+YOLO & 0.41 & 0.65 & 0.19 & 0.08 & 0.61 & 0.08 & 0.83 & 0.69 \\
\hline \multicolumn{9}{|l|}{$K=4$} \\
\hline IlumiCrack(PD4-1)+SSD & 0.43 & 0.62 & 0.17 & 0.11 & 0.68 & 0.04 & 0.74 & 0.70 \\
\hline IlumiCrack(PD4-2)+SSD & 0.32 & 0.58 & 0.002 & 0.11 & 0.55 & 0.17 & 0.78 & 0.68 \\
\hline IlumiCrack(PD4-3)+SSD & 0.04 & 0.14 & 0.0 & 0.0 & 0.001 & 0.0 & 0.15 & 0.02 \\
\hline IlumiCrack(PD4-4)+SSD & 0.37 & 0.39 & 0.82 & 0.16 & 0.69 & 0.63 & 0.68 & 0.73 \\
\hline IlumiCrack(PD4-1)+YOLO & 0.39 & 0.65 & 0.18 & 0.10 & 0.65 & $4 e-4$ & 0.76 & 0.69 \\
\hline IlumiCrack(PD4-2)+ YOLO & 0.34 & 0.54 & 0.07 & 0.15 & 0.50 & 0.15 & 0.76 & 0.63 \\
\hline IlumiCrack(PD4-3)+ YOLO & 0.29 & 0.70 & 0.30 & 0.25 & 0.49 & 0.0 & 0.60 & 0.57 \\
\hline IlumiCrack(PD4-4)+ YOLO & 0.29 & 0.56 & 0.57 & 0.53 & 0.62 & 0.91 & 0.64 & 0.70 \\
\hline
\end{tabular}

In Table 6, we also discuss the overall mAP of the target method with different number of clusters. We can see that the proposed method with two clusters of images with HSV $(K=2)$ outperforms those with three or more clusters of HSV.

Table 6. Overall mAP of the target methods

\begin{tabular}{cc}
\hline Algorithms & mAP \\
\hline SSD & 0.46 \\
\hline K=2 & \\
\hline IlumiCrack (PD2-1\&SSD) & $\mathbf{0 . 5 7}$ \\
IlumiCrack (PD2-2\&SSD) & $\mathbf{0 . 6 5}$ \\
IlumiCrack (PD2-1\&YOLO) & 0.46 \\
IlumiCrack (PD2-2\&YOLO) & 0.55 \\
\hline
\end{tabular}




\begin{aligned} & \hline K=3 \\ & \hline IlumiCrack (PD3-1\&SSD) 0.24 \\ & IlumiCrack (PD3-2\&SSD) 0.49 \\ & IlumiCrack (PD3-3\&SSD) 0.43 \\ & IlumiCrack (PD3-1\&YOLO) 0.34 \\ & IlumiCrack (PD3-2\&YOLO) 0.47 \\ & IlumiCrack (PD3-3\&YOLO) 0.44 \\ & \hline K=4 \\ & \hline IlumiCrack (PD4-1\&SSD) 0.44 \\ & IlumiCrack (PD4-2\&SSD) 0.40 \\ & IlumiCrack (PD4-3\&SSD) 0.04 \\ & IlumiCrack (PD4-1\&YOLO) 0.56 \\ & IlumiCrack (PD4-2\& YOLO) 0.43 \\ & IlumiCrack (PD4-3\& YOLO) 0.39 \\ & IlumiCrack (PD4-4\& YOLO) 0.40 \\ & \hline\end{aligned}

\subsection{Implementation Results}

The experimental results shown in Figs. 13-17. are derived from the IlumiCrack(PD2-1)+SSD and IlumiCrack(PD2-2)+SSD models which outperform the other configurations.

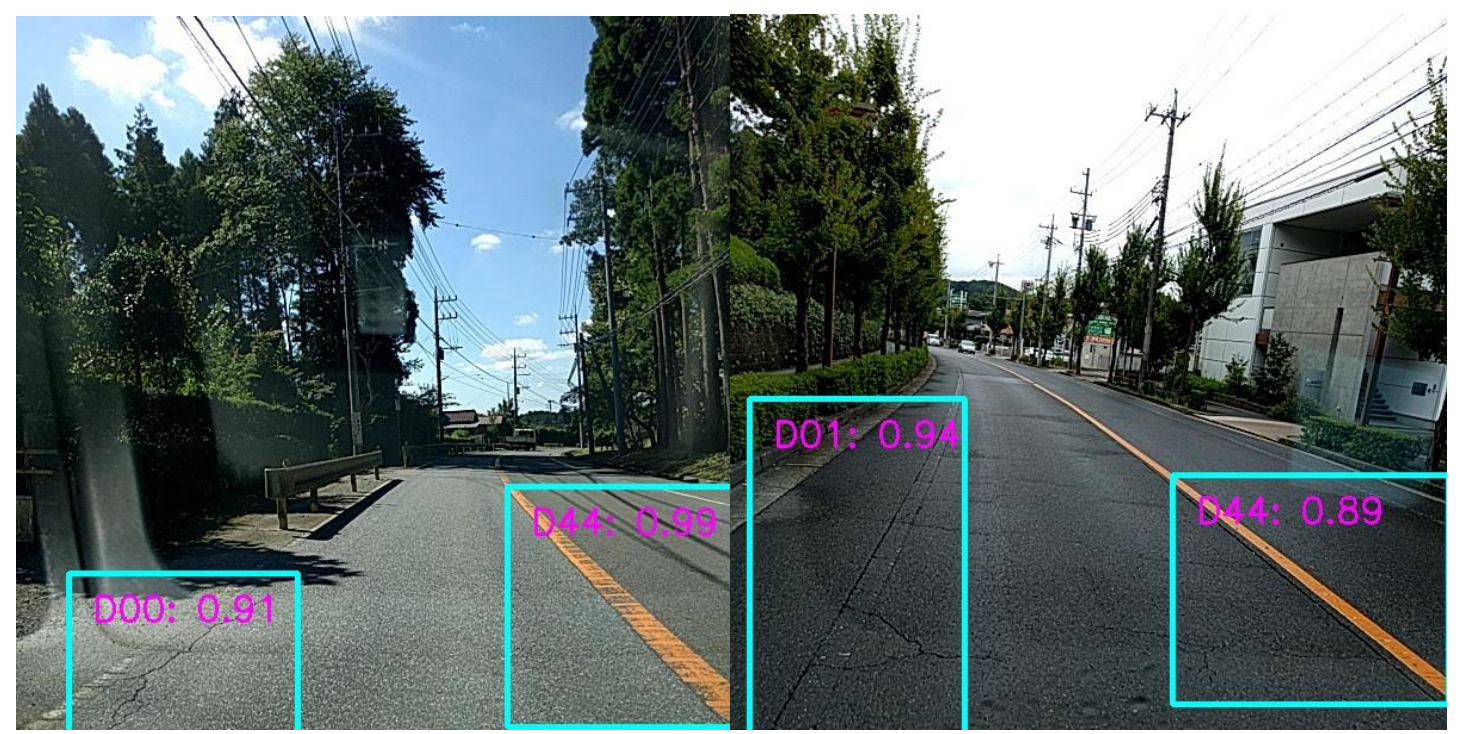

Fig. 13. Object detection results 


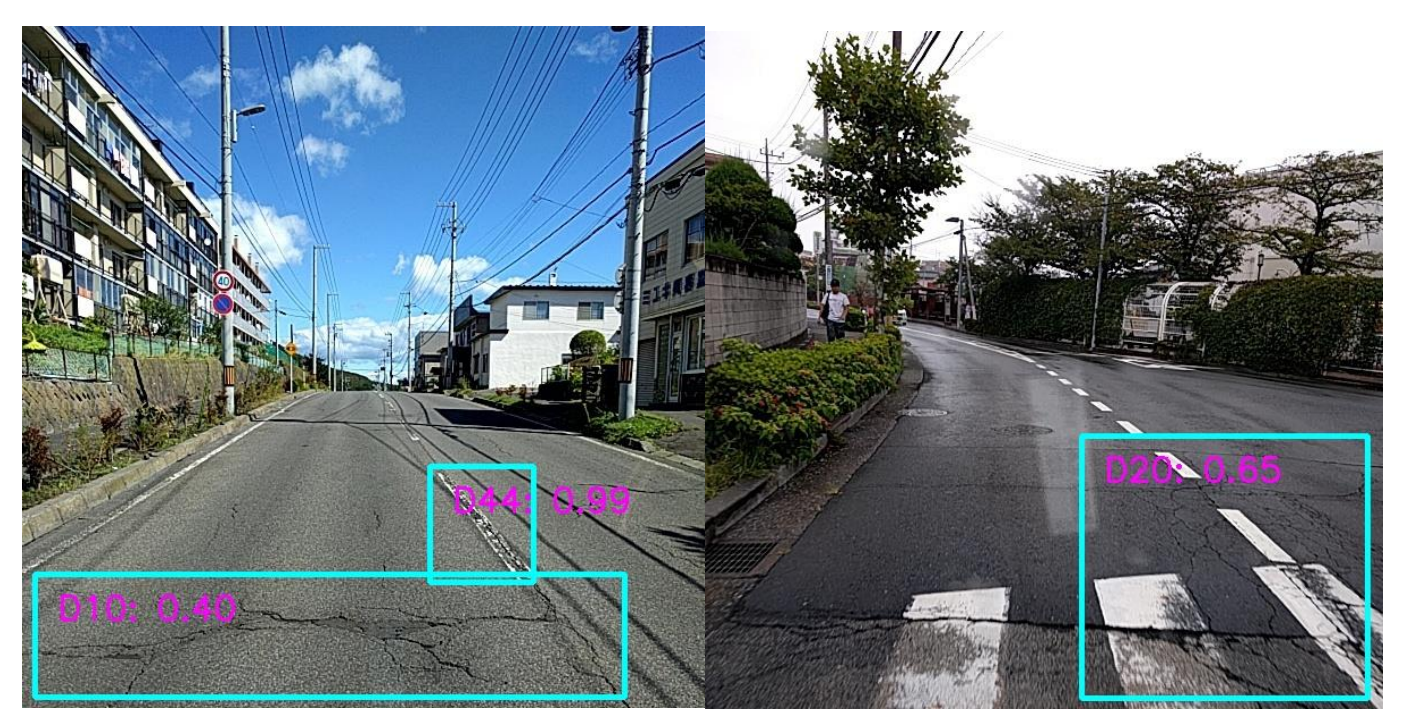

Fig. 14. Object detection results

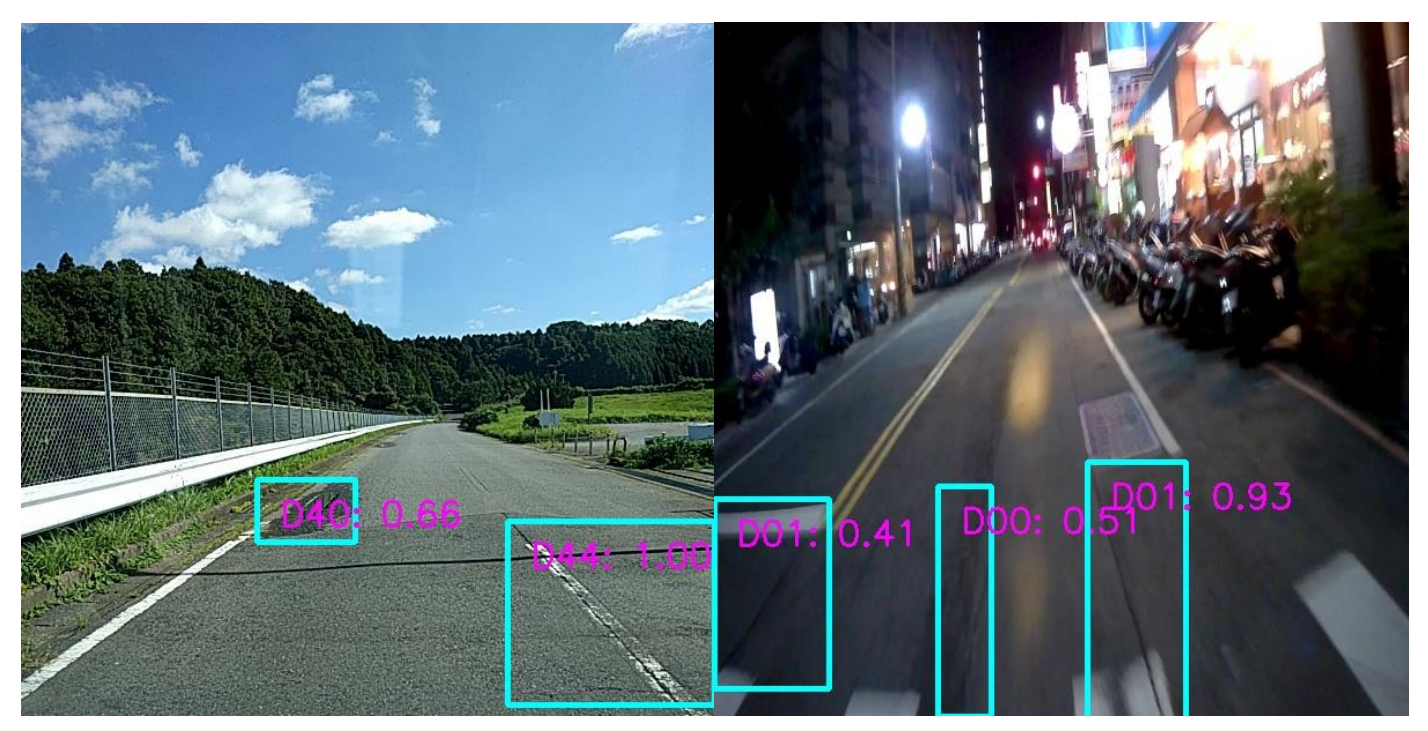

Fig. 15. Object detection results

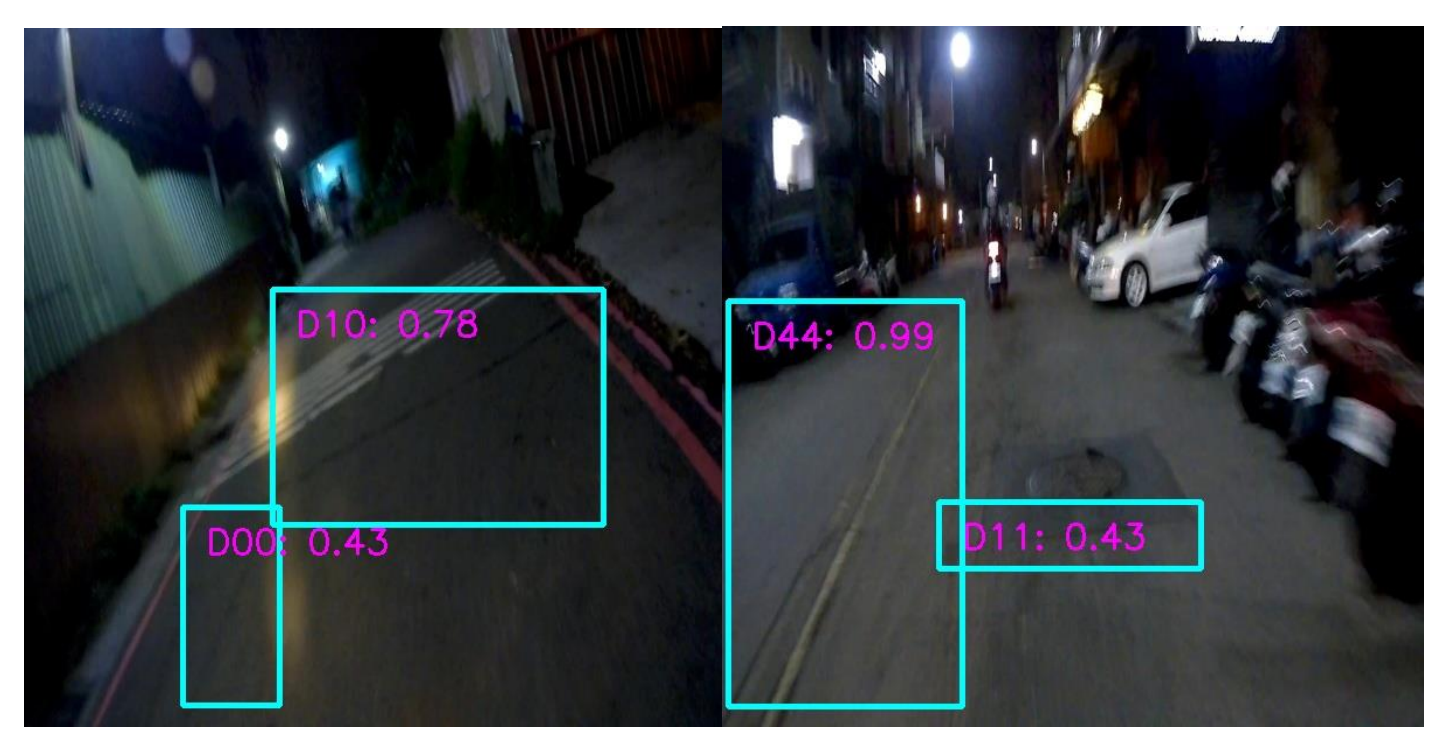

Fig. 16. Object detection results 


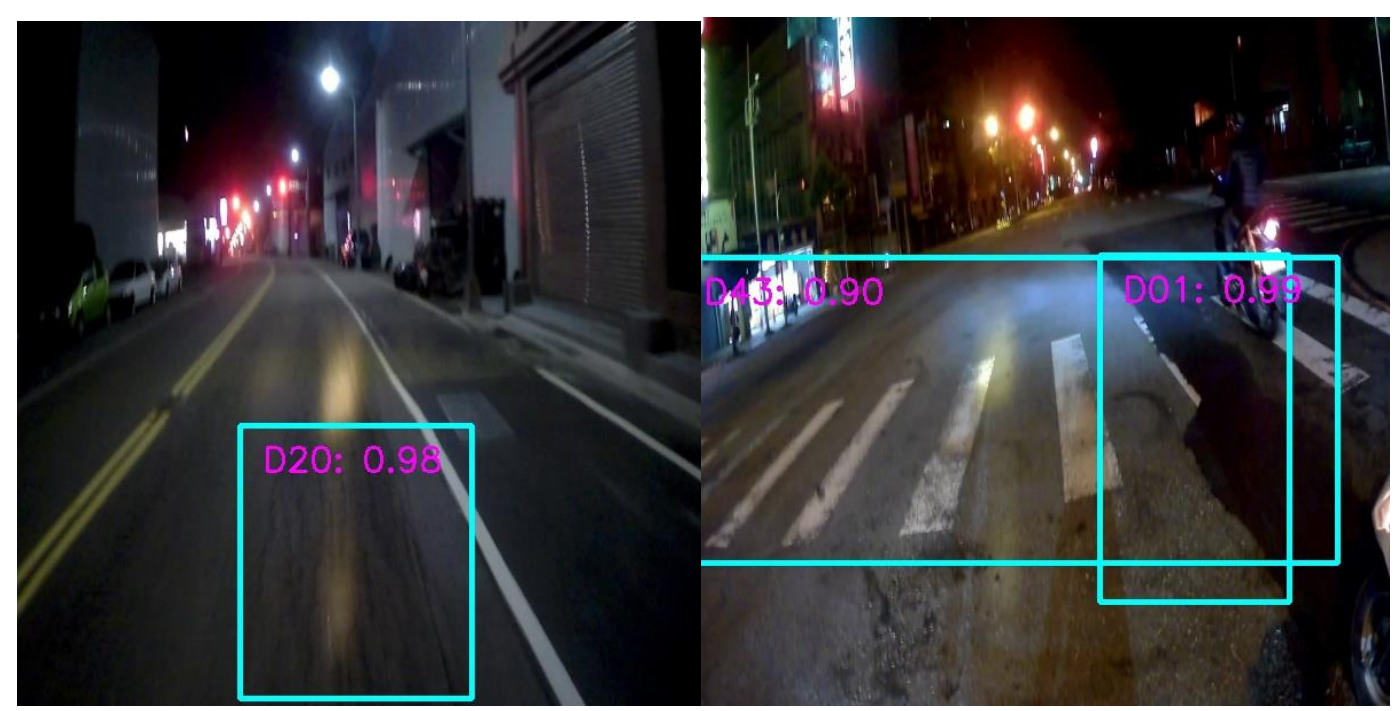

Fig. 17. Object detection results 
4.5. Training Performance

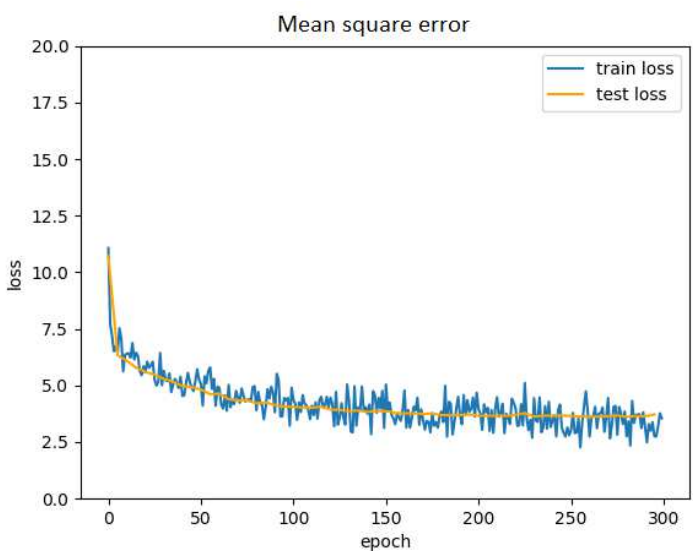

(a) overall

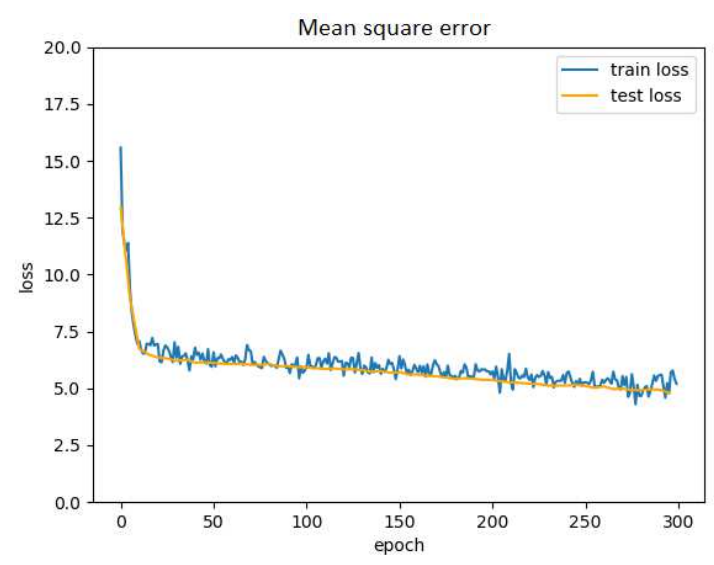

(c) PD2-2

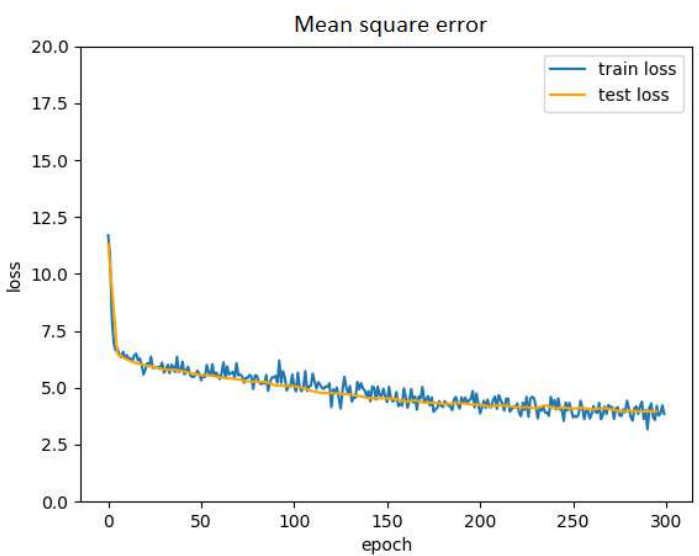

(e) PD3-2

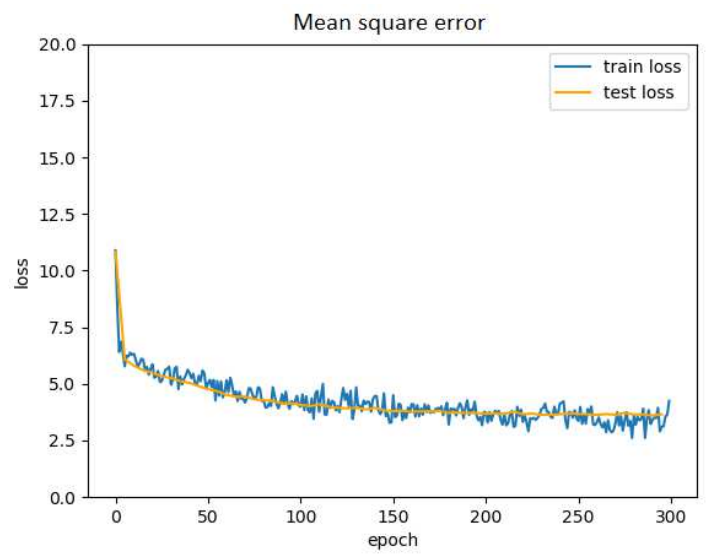

(b) PD2-1

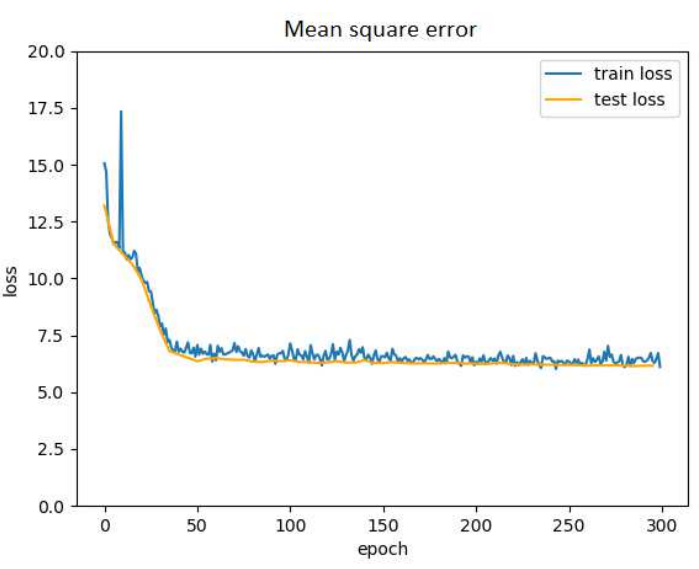

(d) PD3-1

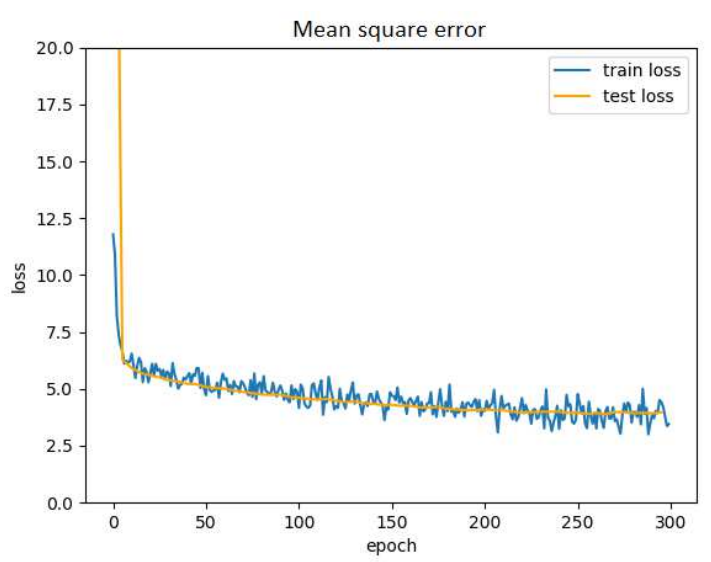

(f) PD3-3

Fig. 18. Training and test performance of datasets using SSD (a) without category, (b) in group 1 with two categories, (c) in group 2 with two categories, (d) in group 1 with three categories, (e) in group 2 with three categories and (f) in group 3 with three categories

This section presents the performance of models under training and test datasets. Figures 18 and 19 present that the training loss and test loss decrease rapidly before 20 epochs based on the SSD framework. In Fig. 18(a), the model without categories produces higher fluctuation in training loss than those models with two and three 
categories (see Fig. 18(b)-(f)). In other words, brightness categories may benefit crack detection models in reducing training loss. However, as shown in Fig. 19, categorizing datasets in four groups produces considerably higher training loss. Therefore, an overcategorized framework may not benefit crack detection due to the limited image patterns or features. Overall, the proposed framework with or without categories has low overfitting because the test losses are decreased smoothly after 10 epochs such that the proposed model has good performance on the test dataset.

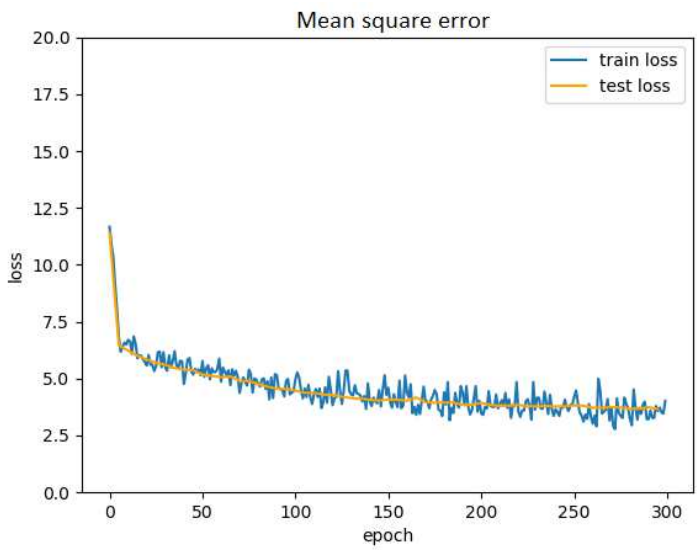

(a) PD4-1

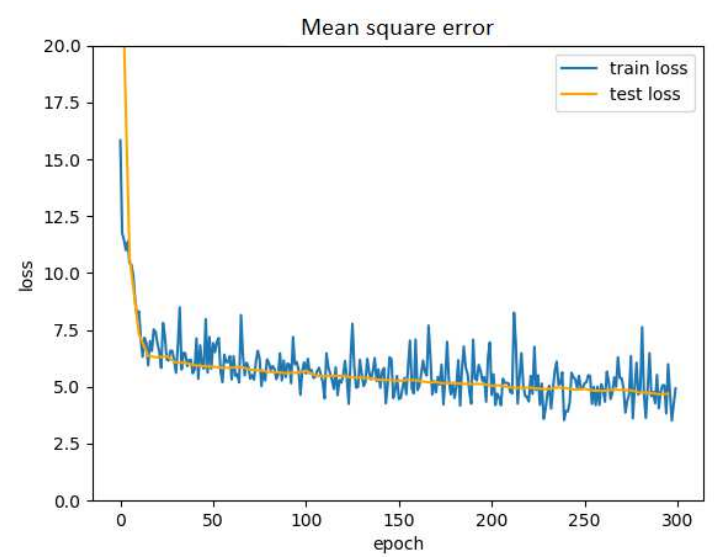

(c) PD4-3

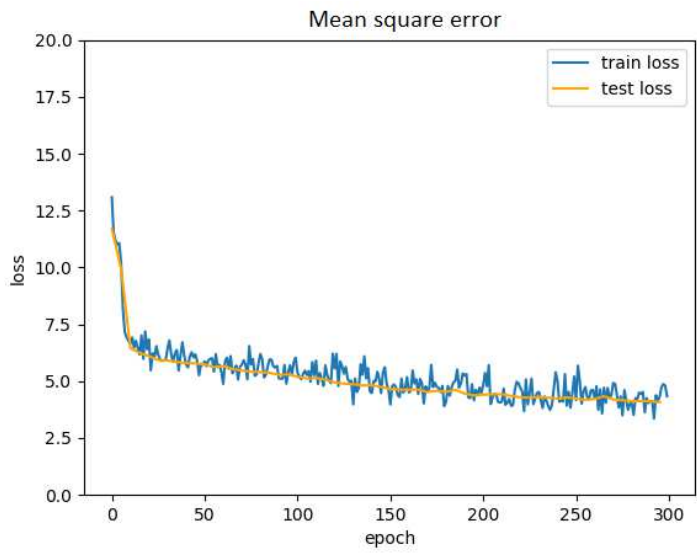

(b) PD4-1

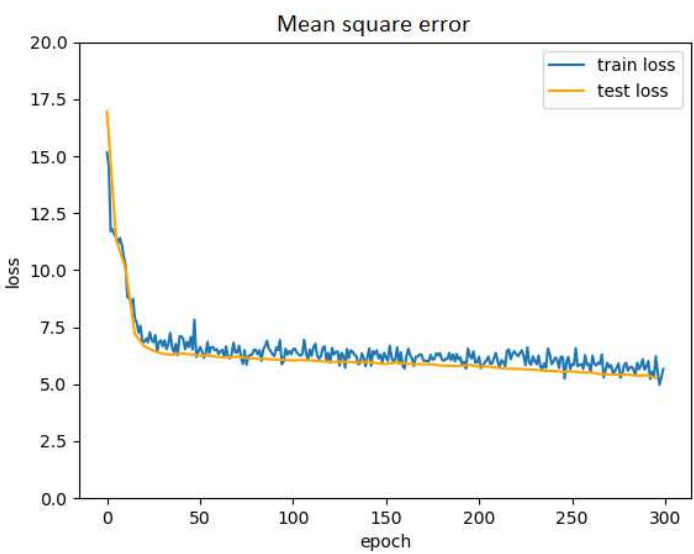

(d) PD4-4

Fig. 19. Training and test performance of datasets in (a) group 1, (b) group 2, (c) group 3 and (d) group 4 with four categories

Figures 20 and 21 respectively show training and test performance for YOLO. In PD2, the training loss fluctuates significantly in a wide spectrum of epochs. In Figs. 20 and 21, PD3 and PD4 present relatively stable training loss compared to PD2. In the figures, overfitting appears after 50 epochs and test loss increases rapidly, indicating that the testing performance of the YOLO framework underperforms the SSD framework for road crack detection. Road surface images are relatively monotonous compared to those of other applications, even though we included a variety of illumination conditions in the dataset. For images with road surfaces in the background, 
the dataset with two categories achieves optimal performance.

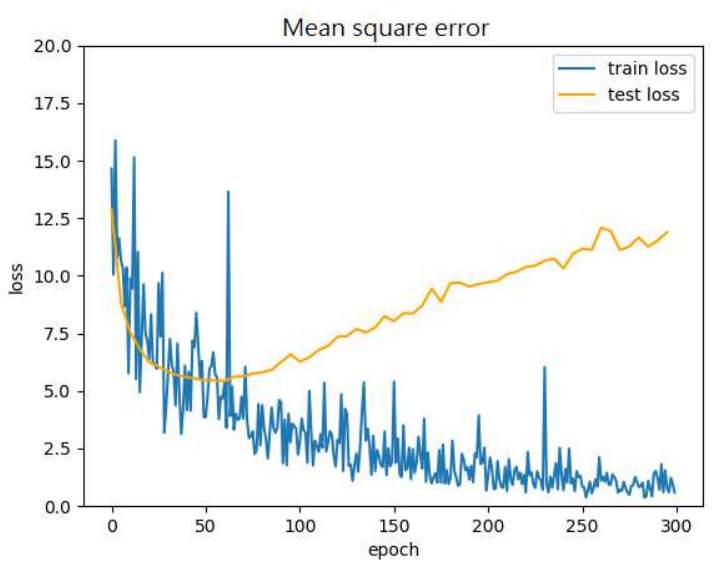

(a) overall

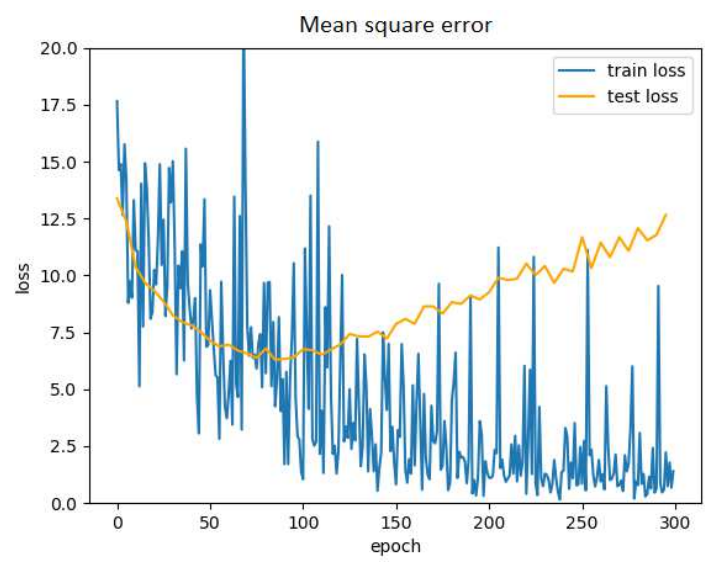

(c) PD2-2

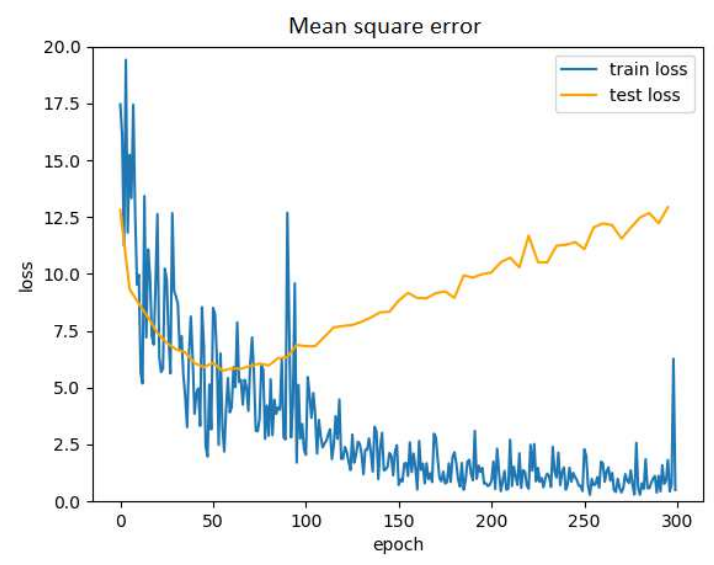

(e) PD3-2

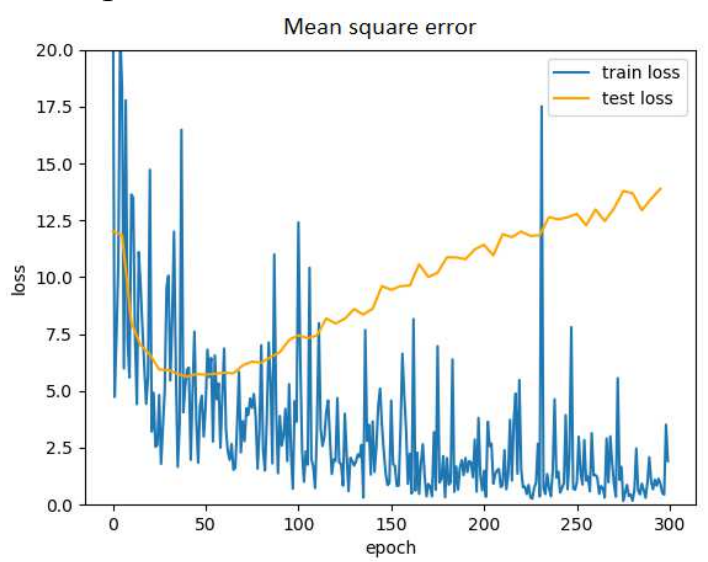

(b) PD2-1

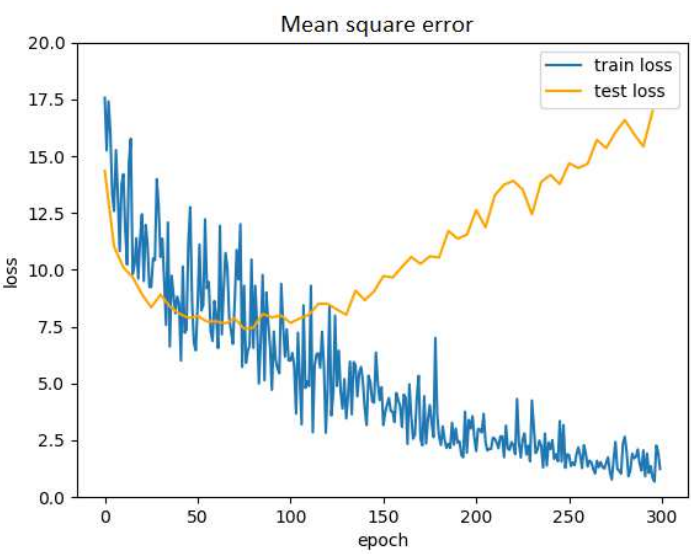

(d) PD3-1

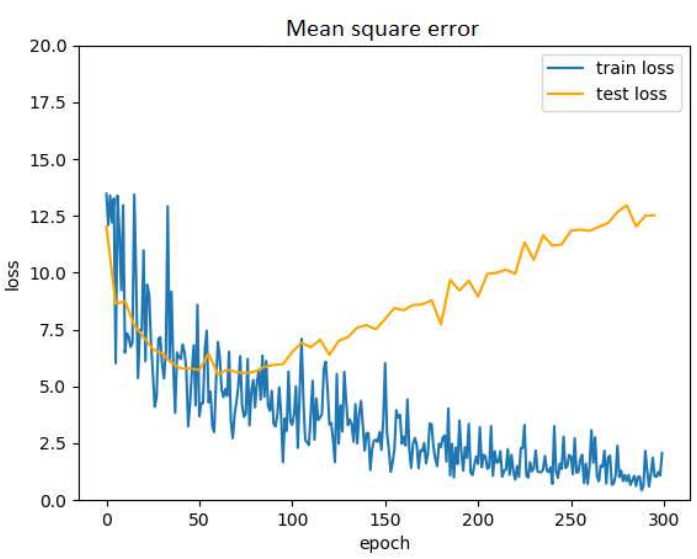

(f) PD3-3

Fig. 20. Training and test performance of datasets using YOLO (a) in group 1 with two categories, (b) in group 2 with two categories, (c) in group 1 with three categories, (d) in group 2 with three categories and (e) in group 3 with three categories 


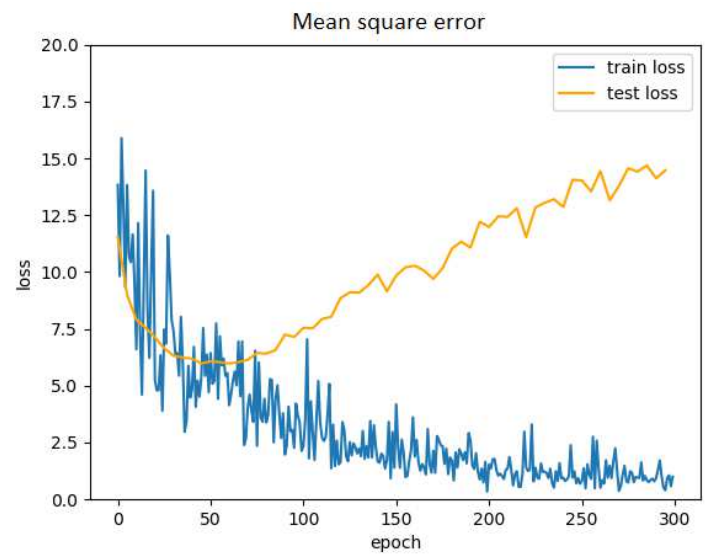

(a) PD4-1

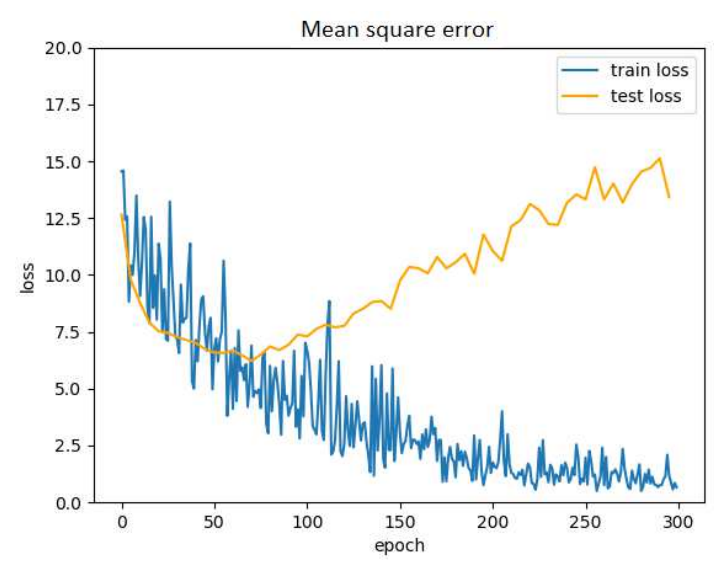

(c) PD4-3

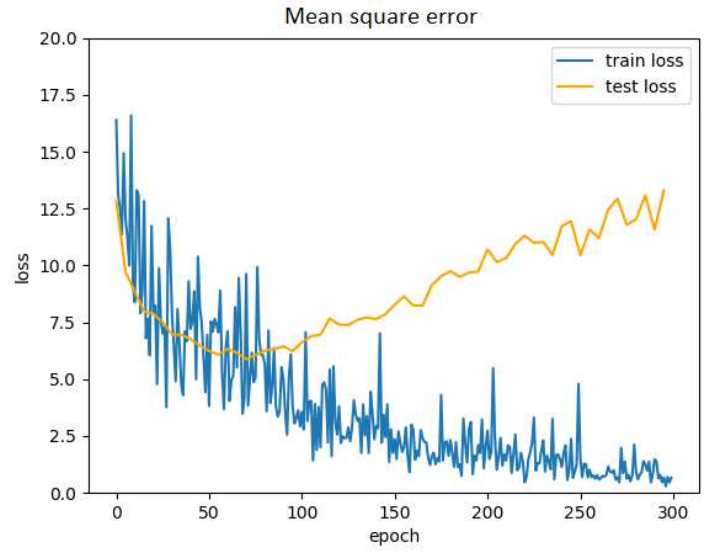

(b) PD4-1

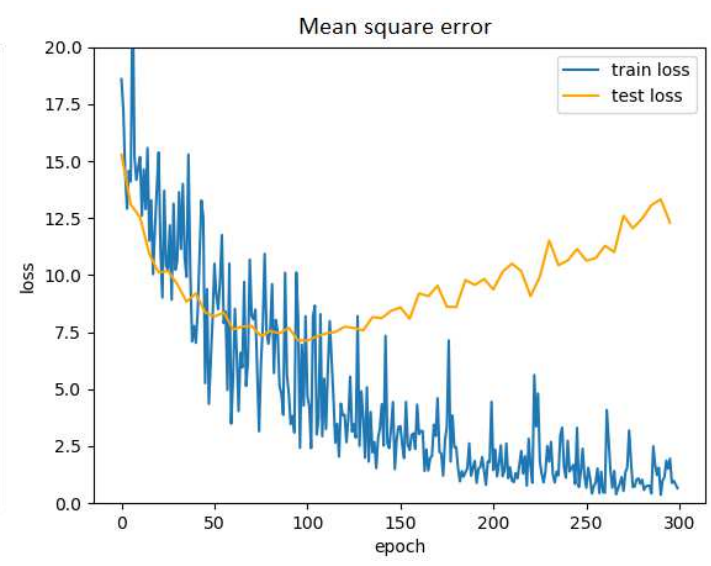

(d) PD4-4

Fig. 21. Training and test performance of datasets using YOLO in (a) group 1, (b) group 2,

(c) group 3 and (d) group 4 with four categories

\section{Conclusions}

Different from previous studies on road crack detection, we focus on detecting cracks under a range of illumination conditions. To achieve this goal, we construct a new largescale dataset of images captured under nighttime or otherwise poorly illuminated conditions. These images were combined with existing datasets of authentic road images, and those images featuring road cracks or similar features were manually confirmed and split into eight classifications by type. The proposed framework initially uses GMM to classify HVS pixels HVS into two, three and four groups, and classifies the dataset images with group proportions (ratio) into two, three and four categories. Using the proposed HSV categories, a dataset with two classes achieves the best precision and recall rate for feature recognition. The proposed framework also outperforms YOLO v3 and SSD for both nighttime and daytime conditions. Based on the results, we believe that pixel classification with HSV values improves automatic road crack detection in environments with varying illumination conditions, while using three or more pixel groups does not improve the precision or recall rates. Integrating 
the proposed method into cloud-based systems will allow road maintenance agencies to automate and outsource crack detection and identification to the general driving public, thus conserving municipal budgets and improving road maintenance efficiency.

\section{Reference}

[1] Protopapadakis, E., Stentoumis, C., Doulamis, N., Doulamis, A., Loupos, K., Makantasis, K., ... \& Amditis, A. (2016). AUTONOMOUS ROBOTIC INSPECTION IN TUNNELS. ISPRS Annals of Photogrammetry, Remote Sensing \& Spatial Information Sciences, 3(5).

[2] Turkan, Y., Hong, J., Laflamme, S., \& Puri, N. (2018). Adaptive wavelet neural network for terrestrial laser scanner-based crack detection. Automation in construction, 94, 191-202.

[3] Sinha, S. K., \& Fieguth, P. W. (2006). Automated detection of cracks in buried concrete pipe images. Automation in Construction, 15(1), 58-72.

[4] Fujita, Y., \& Hamamoto, Y. (2011). A robust automatic crack detection method from noisy concrete surfaces. Machine Vision and Applications, 22(2), 245-254.

[5] He, K., Gkioxari, G., Dollár, P., \& Girshick, R. (2017). Mask r-cnn. In Proceedings of the IEEE international conference on computer vision (pp. 2961-2969).

[6] Hinton, G. E., Osindero, S., \& Teh, Y.-W. (2006). A fast learning algorithm for deep belief nets. Neural computation, 18(7), 1527-1554.

[7] LeCun, Y., Boser, B., Denker, J. S., Henderson, D., Howard, R. E., Hubbard, W., \& Jackel, L. D. J. N. C. (1989). Backpropagation applied to handwritten zip code recognition. 1(4), 541-551.

[8] Elman, J. L. J. C. s. (1990). Finding structure in time. 14(2), 179-211.

[9] Construction bureau of Taichung City government, Citizen Service Hotline, (2015), Retrieved from https://itunes.apple.com/tw/app/\%E8\%87\%BA\%E4\%B8\%AD\%E5\%A5\%BD\%E5\%A5\% BD\%E8\%A1\%8C/id932232922?mt=8

[10] Zalama, E., Gómez-García-Bermejo, J., Medina, R., \& Llamas, J. (2014). Road crack detection using visual features extracted by Gabor filters. Computer-Aided Civil and Infrastructure Engineering, 29(5), 342-358.

[11] Zhang, L., Yang, F., Zhang, Y. D., \& Zhu, Y. J. (2016). Road crack detection using deep convolutional neural network. Paper presented at the Image Processing (ICIP), 2016 IEEE International Conference on.

[12] Maeda, H., Sekimoto, Y., Seto, T., Kashiyama, T., \& Omata, H. (2018). Road damage detection using deep neural networks with images captured through a smartphone. Computer-Aided Civil and Infrastructure Engineering.

[13] Redmon, J., Divvala, S., Girshick, R., \& Farhadi, A. (2016). You only look once: 
Unified, real-time object detection. Paper presented at the Proceedings of the IEEE conference on computer vision and pattern recognition.

[14] Schwenk, H., \& Bengio, Y. (1998). Training methods for adaptive boosting of neural networks. In Advances in neural information processing systems (pp. 647-653).

[15] Dempster, A. P., Laird, N. M., \& Rubin, D. B. J. J. o. t. R. S. S. S. B. (1977). Maximum likelihood from incomplete data via the EM algorithm. 39(1), 1-22.

[16] Gao, X., Boult, T. E., Coetzee, F., \& Ramesh, V. (2000). Error analysis of background adaption. Paper presented at the Proceedings IEEE Conference on Computer Vision and Pattern Recognition. CVPR 2000 (Cat. No. PR00662).

[17] Huang, Y., Englehart, K. B., Hudgins, B., \& Chan, A. D. (2005). A Gaussian mixture model based classification scheme for myoelectric control of powered upper limb prostheses. IEEE Transactions on Biomedical Engineering, 52(11), 1801-1811.

[18] Zeng, S., Huang, R., Wang, H., \& Kang, Z. J. N. (2016). Image retrieval using spatiograms of colors quantized by Gaussian Mixture Models. 171, 673-684.

[19] Girshick, R., Donahue, J., Darrell, T., \& Malik, J. (2014). Rich feature hierarchies for accurate object detection and semantic segmentation. Paper presented at the Proceedings of the IEEE conference on computer vision and pattern recognition.

[20] Felzenszwalb, P. F., \& Huttenlocher, D. P. (2004). Efficient graph-based image segmentation. International Journal of Computer Vision, 59(2), 167-181.

[21] Carlile, B., Delamarter, G., Kinney, P., Marti, A., \& Whitney, B. (2017). Improving deep learning by inverse square root linear units (ISRLUs).

[22] Girshick, R. (2015). Fast r-cnn. Paper presented at the Proceedings of the IEEE international conference on computer vision.

[23] Ren, S., He, K., Girshick, R., \& Sun, J. (2015). Faster r-cnn: Towards real-time object detection with region proposal networks. Paper presented at the Advances in neural information processing systems.

[24] Redmon, J. (2016). Darknet: Open Source Neural Networks in C. Retrieved from http://pjreddie.com/darknet/

[25] Redmon, J., \& Farhadi, A. (2017). YOLO9000: better, faster, stronger. Paper presented at the Proceedings of the IEEE conference on computer vision and pattern recognition.

[26] Redmon, J., \& Farhadi, A. J. a. p. a. (2018). Yolov3: An incremental improvement. [27] Lin, T.-Y., Dollár, P., Girshick, R., He, K., Hariharan, B., \& Belongie, S. (2017). Feature pyramid networks for object detection. Paper presented at the Proceedings of the IEEE conference on computer vision and pattern recognition.

[28] He, K., Zhang, X., Ren, S., \& Sun, J. (2016). Deep residual learning for image recognition. Paper presented at the Proceedings of the IEEE conference on computer vision and pattern recognition.

[29] Liu, W., Anguelov, D., Erhan, D., Szegedy, C., Reed, S., Fu, C.-Y., \& Berg, A. C. (2016). 
Ssd: Single shot multibox detector. Paper presented at the European conference on computer vision.

[30] Simonyan, K., \& Zisserman, A. J. a. p. a. (2014). Very deep convolutional networks for large-scale image recognition.

[31] Chen, L.-C., Papandreou, G., Kokkinos, I., Murphy, K., Yuille, A. L. J. I. t. o. p. a., \& intelligence, m. (2017). Deeplab: Semantic image segmentation with deep convolutional nets, atrous convolution, and fully connected crfs. 40(4), 834-848.

[32] Rowl1ng. (2018). Detectron 代码分析：从 Faster R-CNN 到 Mask R-CNN. Retrieved from https://rowl1ng.com/blog/tech/faster-rcnn.html

[33] Saha, B., Davies, D., \& Raghavan, A. (2016). Day night classification of images using thresholding on HSV histogram. In: Google Patents.

[34] Shi, Y., Cui, L., Qi, Z., Meng, F., \& Chen, Z. (2016). Automatic road crack detection using random structured forests. IEEE Transactions on Intelligent Transportation Systems, 17(12), 3434-3445.

[35] Everingham, M., Van Gool, L., Williams, C. K., Winn, J., \& Zisserman, A. J. I. j. o. c. v. (2010). The pascal visual object classes (voc) challenge. 88(2), 303-338. 